\title{
Integrated Power and Attitude Control Design of Satellites Based on a Fuzzy Adaptive Disturbance Observer Using Variable-Speed Control Moment Gyros
}

\author{
Zhongyi Chu ${ }^{1}$ and Jing $\mathrm{Cui}^{2}$ \\ ${ }^{1}$ School of Instrument Science and Opto-Electronic Engineering, Beihang University, Beijing 100191, China \\ ${ }^{2}$ School of Mechanical Engineering and Applied Electronics, Beijing University of Technology, Beijing 101100, China \\ Correspondence should be addressed to Zhongyi Chu; chuzystar@gmail.com
}

Received 1 December 2015; Revised 5 February 2016; Accepted 17 February 2016

Academic Editor: Paolo Tortora

Copyright (C) 2016 Z. Chu and J. Cui. This is an open access article distributed under the Creative Commons Attribution License, which permits unrestricted use, distribution, and reproduction in any medium, provided the original work is properly cited.

To satisfy the requirements for small satellites that seek agile slewing with peak power, this paper investigates integrated power and attitude control using variable-speed control moment gyros (VSCMGs) that consider the mass and inertia of gimbals and wheels. The paper also details the process for developing the controller by considering various environments in which the controller may be implemented. A fuzzy adaptive disturbance observer (FADO) is proposed to estimate and compensate for the effects of equivalent disturbances. The algorithms can simultaneously track attitude and power. The simulation results illustrate the effectiveness of the control approach, which exhibits an improvement of 80 percent compared with alternate approaches that do not employ a FADO.

\section{Introduction}

The concept of an attitude system and energy storage was first proposed in 1961 and has become particularly popular over the last two decades. However, significant challenges must be addressed before this well-documented concept can be realized for operational satellite missions [1]; specifically, flywheels are typically used for onboard orbiting satellites to control the attitude, and thus, a suitable algorithm must be developed to meet both the attitude and power requirements. Over the last two decades, many researchers have investigated this topic using momentum wheels or variable-speed control moment gyros (VSCMGs). The VSCMG, which combines a typical control moment gyroscope and a reaction wheel, is a momentum exchange device that is capable of providing both large control torque for aggressive slew maneuver and small control torque to fine-tune the spacecraft attitude [2]. This dual capability will be beneficial for future small-satellite missions that seek agile slewing with high peak power [3].

Conventional controllers designed for the integrated power and attitude control (IPAC) problem typically employ the linearized equations of motion. However, applied spacecraft, such as those used in satellite surveillance and precision Earth imaging, often involve highly accurate slewing and pointing maneuvers that require the spacecraft to rotate along a relatively large-angle amplitude trajectory. These characteristics necessitate the use of a nonlinear controller design. Using a classical dynamics approach, the nonlinear equations for a VSCMG cluster in a rigid spacecraft have been derived for different kinematic descriptions of the spacecraft orientation. Tsiotras published a series of papers related to this problem $[4,5]$, and Yoon and Tsiotras [4] extended their earlier IPAC developments to include actuation and energy storage with VSCMGs and designed a model-based controller to achieve exact attitude tracking with a perfect known spacecraft model. However, the mass moment of the inertia matrix must be precisely known, and many researchers simplify the model by assuming that the gimbals have zero inertia to facilitate the controller design [6].

In practical situations, the controller design is further complicated by the uncertainties of the spacecraft mass and inertia properties due to fuel consumption, variations in 
the payload, and the deployment of appendages. To address these challenges, an adaptive control scheme is typically chosen for precise attitude tracking control. An adaptive full-state feedback controller without velocity measurement was proposed to compensate for parameter uncertainty [7]. An adaptive law combined with an extended state observer was developed for the attitude tracking of a spacecraft with bounded disturbances [8]. A passivity-based adaptive attitude controller was designed for the IPAC problem of a rigid spacecraft with unknown inertia properties [9]. A nonlinear controller was designed for large rotational maneuvers of a dual-body spacecraft with VSCMGs based on Lyapunov's direct method [10]. Park presented a nonlinear feedback controller for the IPAC problem using magnetically suspended VSCMGs in the presence of system modeling error and flywheel mass imbalance disturbances [11]. An adaptive nonlinear control law based on the Lyapunov theorem was proposed for a system with a VSCMG cluster and uncertain spacecraft inertia properties [12]. However, adaptive control approaches have two major drawbacks [13]. First, the uncertain nonlinearity must satisfy the assumption of "linearity in the parameters," and "regressive matrices" must be determined a priori. The second drawback is the lack of robustness to unmodeled dynamics and external disturbances. Uncertain gimbal friction, actuator disturbances, and nonlinear disturbance torques do not satisfy the linear parameter assumption, and hence, the robustness of a control system against unmodeled dynamics and external disturbances is an important consideration when assessing controller performance.

Several of the available methods do not suffer from the two drawbacks of adaptive control approaches, such as the uncertainty and disturbance estimator (UDE). However, a drawback of the UDE is that it generates large initial control, which may cause the actuators to become saturated, thereby hindering the implementation of the control [14]. Other classical solutions include neural networks (NNs) and/or fuzzy logic systems (FLSs), which can approximate well-defined functions over a compact set to an arbitrary level of accuracy. Recently, Zou et al. [13] proposed an output feedback attitude controller for spacecraft in the presence of structured and unstructured uncertainties based on Chebyshev NNs. An NN-based adaptive attitude tracking controller in the presence of inertia and CMG actuator uncertainties was also developed in [15]. However, the implementation of these controllers requires tedious analyses and high computation complexity.

A disturbance observer- (DOB-) based controller is a practical method of addressing internal parameter uncertainty and external disturbance [16]. However, the traditional DOB-based controller is based on the linear theory and is essentially a robust controller [17]. This paper suggests a new DOB-based controller for IPAC in satellites with VSCMGs in the presence of model uncertainties and external disturbances. The proposed method is based on an adaptive fuzzy disturbance observer (FADO) [18]. Adaptive fuzzy systems are employed to approximate the nonlinear and uncertain terms in the VSCMGs and satellite dynamics. Compared with previous studies, the proposed controller can overcome not only parametric uncertainties, which can also be addressed by the adaptive method, but also nonparametric disturbances, which cannot be addressed by the traditional adaptive method. The proposed controller can achieve asymptotic attitude tracking with an uncertain inertia matrix and guarantee the bound of the tracking errors with external disturbances. Furthermore, the proposed controller does not involve the tedious repressor analyses required by traditional NN-based adaptive controllers and is thus computationally efficient and practical.

To satisfy the requirements of attitude and power for future small-satellite missions that seek agile slewing and high peak power, this paper investigates integrated power and attitude control using VSCMGs, which consider the mass and inertia of the gimbals and wheels, and details the process for developing the controller by considering various environments in which the controller may be implemented. The remainder of this paper is organized as follows: In Section 2, the dynamics model of the maneuvering satellite with VSCMGs is reviewed. In Section 3, the control scheme incorporating a FADO is developed. In Section 4, a computer simulation is performed to demonstrate the effectiveness and applicability of the proposed method. Finally, some conclusions are drawn in Section 5.

\section{Problem Description}

Considering a rigid spacecraft with a cluster of $N$ singlegimbal VSCMGs used to provide internal torques, the attitude dynamics of the spacecraft can be expressed as [4]

$$
\dot{\mathrm{J}} \boldsymbol{\omega}+\mathrm{J} \dot{\boldsymbol{\omega}}+\dot{\mathbf{h}}_{c}=-\left[\boldsymbol{\omega}^{\times}\right] \mathbf{h}+\mathbf{g}_{e},
$$

where the total angular momentum $\mathbf{h}=\mathbf{J} \boldsymbol{\omega}+\mathbf{h}_{c}=\mathbf{J} \boldsymbol{\omega}+$ $\mathbf{A}_{g} \mathbf{I}_{c g} \dot{\boldsymbol{\gamma}}+\mathbf{A}_{s} \mathbf{I}_{w s} \boldsymbol{\Omega}, \boldsymbol{\gamma}=\left(\gamma_{1}, \ldots, \gamma_{N}\right)^{T}$, and $\boldsymbol{\Omega}=\left(\Omega_{1}, \ldots, \Omega_{N}\right)^{T}$ are column vectors whose elements are the gimbal angles and wheel speeds of the VSCMGs with respect to the gimbals, respectively. $\mathbf{g}_{e}$ is an external torque vector. For any vector $\mathbf{x}=$ $\left(x_{1}, x_{2}, x_{3}\right)^{T}$, the notation $\left[\mathbf{x}^{\times}\right]$denotes the skew-symmetric matrix

$$
\mathbf{x}^{\times}=\left[\begin{array}{ccc}
0 & -x_{3} & x_{2} \\
x_{3} & 0 & -x_{1} \\
-x_{2} & x_{1} & 0
\end{array}\right] .
$$

The matrix $\mathbf{J}$ is the inertia matrix of the entire spacecraft, $\mathbf{J}=\mathbf{J}_{0}+\mathbf{A}_{s} \mathbf{I}_{c s} \mathbf{A}_{s}^{T}+\mathbf{A}_{t} \mathbf{I}_{c t} \mathbf{A}_{t}^{T}+\mathbf{A}_{g} \mathbf{I}_{c g} \mathbf{A}_{g}^{T}$, where $\mathbf{J}_{0}$ is the combined matrix of the inertia matrix of the spacecraft platform and the point masses of the VSCMGs. Specifically $\mathbf{I}_{c *}=\mathbf{I}_{g^{*}}+\mathbf{I}_{w *}, \mathbf{I}_{g^{*}}=\operatorname{diag}\left(I_{c * 1}, \ldots, I_{c * N}\right)$ and $\mathbf{I}_{w *}=$ $\operatorname{diag}\left(I_{w * 1}, \ldots, I_{w * N}\right)$, where $*$ is $\mathbf{g}$, $\mathbf{s}$, or $\mathbf{t}$. The matrices $\mathbf{A}_{*}=$ $\left(e_{* 1}, \ldots, e_{* N}\right)$ have the gimbal $(\mathbf{g})$, spin $(\mathbf{s})$, and transverse (t) directional unit vectors expressed in the body frame as columns, where $e_{* N}$ is the unit column vector for the $j$ th VSCMG along the direction of the gimbal, spin, or transverse 
axis. Furthermore, $\mathbf{A}_{s}=\mathbf{A}_{s}(\gamma)$ and $\mathbf{A}_{t}=\mathbf{A}_{t}(\boldsymbol{\gamma})$, which can be written using their initial values at time $t=0$ as follows:

$$
\begin{aligned}
& \mathbf{A}_{g}=\mathbf{A}_{g 0}, \\
& \mathbf{A}_{s}=\mathbf{A}_{s 0}[\cos \gamma]^{d}+\mathbf{A}_{t 0}[\sin \gamma]^{d}, \\
& \mathbf{A}_{t}=\mathbf{A}_{t 0}[\cos \gamma]^{d}-\mathbf{A}_{s 0}[\sin \gamma]^{d},
\end{aligned}
$$

where $[\cos \gamma]^{d}=\operatorname{diag}\left(\cos \gamma_{1}, \ldots, \cos \gamma_{N}\right)$ and $[\sin \gamma]^{d}=$ $\operatorname{diag}\left(\sin \gamma_{1}, \ldots \sin \gamma_{N}\right)$.

The so-called modified Rodrigues parameters (MRPs) are used to describe the attitude kinematics error of the spacecraft. The MRPs have the advantages of being well defined for the entire range of rotations, and the differential equation that governs the kinematics in terms of the MRPs is given by

$$
\dot{\sigma}=\mathbf{G}(\boldsymbol{\sigma}) \boldsymbol{\omega}
$$

where

$$
\mathbf{G}(\boldsymbol{\sigma})=\frac{1}{2}\left\{\mathbf{I}+\left[\boldsymbol{\sigma}^{\times}\right]+\boldsymbol{\sigma} \boldsymbol{\sigma}^{T}-\left[\frac{1}{2}\left(1+\boldsymbol{\sigma}^{T} \boldsymbol{\sigma}\right)\right] \mathbf{I}\right\}
$$

and $\mathbf{I}$ is the identity matrix. Let $\mathbf{h}_{1}=\mathbf{J} \boldsymbol{\omega}$ and $\mathbf{h}_{2}=\mathbf{A}_{s} \mathbf{I}_{w s} \mathbf{\Omega}$ and assume that the term $\ddot{\gamma}$ can be neglected. Then, the nominal dynamics equation of the system can be written in standard form [4]

$$
\mathbf{H}(\boldsymbol{\sigma}) \ddot{\boldsymbol{\sigma}}+\mathbf{C}(\boldsymbol{\sigma}, \dot{\boldsymbol{\sigma}}) \dot{\boldsymbol{\sigma}}=\mathbf{F},
$$

where

$$
\begin{aligned}
& \mathbf{H}(\boldsymbol{\sigma})=\mathbf{G}^{-T}(\boldsymbol{\sigma}) \mathbf{J G}^{-1}(\boldsymbol{\sigma}), \\
& \mathbf{C}(\boldsymbol{\sigma}, \dot{\boldsymbol{\sigma}})=-\mathbf{G}^{-T}(\boldsymbol{\sigma}) \mathbf{J G}^{-1}(\boldsymbol{\sigma}) \dot{\mathbf{G}}(\boldsymbol{\sigma}, \dot{\boldsymbol{\sigma}}) \mathbf{G}^{-1}(\boldsymbol{\sigma}) \\
& \quad-\mathbf{G}^{-T}(\boldsymbol{\sigma})\left[\mathbf{h}_{1}^{\times}\right] \mathbf{G}^{-1}(\boldsymbol{\sigma}), \\
& \mathbf{F}=\mathbf{G}^{-T}(\boldsymbol{\sigma})\left[\mathbf{h}_{2}^{\times}\right] \boldsymbol{\omega}-\mathbf{G}^{-T}(\boldsymbol{\sigma}) \mathbf{C} \mathbf{t}-\frac{1}{2} \mathbf{G}^{-T}(\boldsymbol{\sigma}) \dot{\mathbf{J}} \boldsymbol{\omega}, \\
& \mathbf{C} \mathbf{t}=\mathbf{C}^{*} \dot{\boldsymbol{\gamma}}+\mathbf{D} \dot{\boldsymbol{\Omega}}, \\
& \mathbf{C}^{*}=\mathbf{A}_{t} \mathbf{I}_{w s}[\mathbf{\Omega}]^{d}+\left[\boldsymbol{\omega}^{\times}\right] \mathbf{A}_{g} \mathbf{I}_{c g} \\
& \quad+\frac{1}{2}\left[\left(e_{s 1} e_{t 1}^{T}+e_{t 1} e_{s 1}^{T}\right) \boldsymbol{\omega}, \ldots,\left(e_{s N} e_{t N}^{T}+e_{t N} e_{s N}^{T}\right) \boldsymbol{\omega}\right] \\
& \quad \cdot\left(\mathbf{I}_{c s}-\mathbf{I}_{c t}\right), \\
& \mathbf{D}=\mathbf{A}_{s} \mathbf{I}_{w s} .
\end{aligned}
$$

The matrix $\dot{\mathbf{H}}(\boldsymbol{\sigma})-2 \mathbf{C}(\boldsymbol{\sigma}, \dot{\boldsymbol{\sigma}})-\mathbf{G}^{-T} \dot{J G}^{-1}$ is skew symmetric, and the term $\dot{\mathbf{G}}(\boldsymbol{\sigma}, \dot{\boldsymbol{\sigma}})$ can be derived by differentiating (5) as

$$
\dot{\mathbf{G}}(\boldsymbol{\sigma}, \dot{\boldsymbol{\sigma}})=\frac{1}{2}\left(\left[\dot{\boldsymbol{\sigma}}^{\times}\right]+\dot{\boldsymbol{\sigma}} \boldsymbol{\sigma}^{T}+\boldsymbol{\sigma} \dot{\boldsymbol{\sigma}}^{T}-\dot{\boldsymbol{\sigma}}^{T} \boldsymbol{\sigma} \mathbf{I}\right) .
$$

The actual values are assumed to be different from the nominal values due to uncertainties in the inertia, dynamic actuator friction effects, and nonlinear disturbances.
The inertial momentum of the spacecraft and VSCMGs cannot be obtained precisely. However, the actual parameter can be expressed as the sum of the nominal value and the uncertainty value as follows:

$$
\begin{gathered}
\breve{\mathbf{J}}_{0}=\mathbf{J}_{0}+\Delta \mathbf{J}_{0}, \\
\breve{\mathbf{I}}_{c s}=\mathbf{I}_{c s}+\Delta \mathbf{I}_{c s}, \\
\breve{\mathbf{I}}_{c t}=\mathbf{I}_{c t}+\Delta \mathbf{I}_{c t}, \\
\breve{\mathbf{I}}_{c g}=\mathbf{I}_{c g}+\Delta \mathbf{I}_{c g}, \\
\breve{\mathbf{I}}_{w s}=\mathbf{I}_{w s}+\Delta \mathbf{I}_{w s}, \\
\breve{\mathbf{I}}_{w t}=\mathbf{I}_{w t}+\Delta \mathbf{I}_{w t}, \\
\breve{\mathbf{I}}_{w g}=\mathbf{I}_{w g}+\Delta \mathbf{I}_{w g} .
\end{gathered}
$$

The presence of dynamic uncertainty and static friction in the VSCMG gimbals are expressed as

$$
\breve{\mathbf{C}} \mathbf{t}=\mathbf{C} \mathbf{t}+\mathbf{F}_{d} \dot{\gamma}+\mathbf{F}_{s} \operatorname{sgn}(\gamma) .
$$

Initial uncertainty in the transformation matrices $\mathbf{A}_{t 0}(\gamma)$ and $\mathbf{A}_{s 0}(\gamma)$ exists and is expressed as

$$
\begin{aligned}
& \breve{\mathbf{A}}_{s 0}=\mathbf{A}_{s 0}+\Delta \mathbf{A}_{s 0}, \\
& \breve{\mathbf{A}}_{t 0}=\mathbf{A}_{t 0}+\Delta \mathbf{A}_{t 0},
\end{aligned}
$$

where $\breve{x}$ represents the actual value of variable $x$.

Substituting (9)-(11) into (6) and including the external disturbance $\mathbf{g}_{e}$, the actual dynamics of the model can be expressed as

$$
\breve{\mathbf{H}}(\boldsymbol{\sigma}) \ddot{\boldsymbol{\sigma}}+\breve{\mathbf{C}}(\boldsymbol{\sigma}, \dot{\boldsymbol{\sigma}}) \dot{\boldsymbol{\sigma}}+\mathbf{g}_{e}=\breve{\mathbf{F}},
$$

where

$$
\begin{aligned}
\breve{\mathbf{H}}(\boldsymbol{\sigma})= & \mathbf{G}^{-T}(\boldsymbol{\sigma}) \breve{\mathbf{J}}^{-1}(\boldsymbol{\sigma}), \\
\breve{\mathbf{C}}(\boldsymbol{\sigma}, \dot{\boldsymbol{\sigma}})= & -\mathbf{G}^{-T}(\boldsymbol{\sigma}) \breve{\mathbf{J}} \mathbf{G}^{-1}(\boldsymbol{\sigma}) \dot{\mathbf{G}}(\boldsymbol{\sigma}, \dot{\boldsymbol{\sigma}}) \mathbf{G}^{-1}(\boldsymbol{\sigma}) \\
& -\mathbf{G}^{-T}(\boldsymbol{\sigma})\left[\breve{\mathbf{h}}_{1}^{\times}\right] \mathbf{G}^{-1}(\boldsymbol{\sigma}), \\
\breve{\mathbf{F}}= & \mathbf{G}^{-T}(\boldsymbol{\sigma})\left[\breve{\mathbf{h}}_{2}^{\times}\right] \boldsymbol{\omega}-\mathbf{G}^{-T}(\boldsymbol{\sigma}) \breve{\mathbf{C}} \mathbf{t} \\
& -\frac{1}{2} \mathbf{G}^{-T}(\boldsymbol{\sigma}) \dot{\mathbf{J}} \omega .
\end{aligned}
$$

Based on the nominal model, the actual model of (12) can be rewritten as

$$
\mathbf{H}(\boldsymbol{\sigma}) \ddot{\boldsymbol{\sigma}}+\mathbf{C}(\boldsymbol{\sigma}, \dot{\boldsymbol{\sigma}}) \dot{\boldsymbol{\sigma}}+\mathbf{d}(\boldsymbol{\sigma}, \dot{\boldsymbol{\sigma}}, \dot{\gamma}, \dot{\boldsymbol{\Omega}}, \ldots)=\mathbf{F},
$$

where

$$
\begin{aligned}
\mathbf{d}(\boldsymbol{\sigma}, \dot{\boldsymbol{\sigma}}, \dot{\boldsymbol{\gamma}}, \dot{\boldsymbol{\Omega}}, \ldots) \\
=-\mathbf{H}(\boldsymbol{\sigma}) \breve{\mathbf{H}}(\boldsymbol{\sigma})\left(\breve{\mathbf{F}}-\mathbf{g}_{e}-\breve{\mathbf{C}}(\boldsymbol{\sigma}, \dot{\boldsymbol{\sigma}}) \dot{\boldsymbol{\sigma}}\right) \\
\quad+(\mathbf{F}-\mathbf{C}(\boldsymbol{\sigma}, \dot{\boldsymbol{\sigma}}) \dot{\boldsymbol{\sigma}})
\end{aligned}
$$


denotes the uncertainties and external disturbances of the system. By combining the uncertainties and external disturbance, the dynamics of the system can be expressed as

$$
\begin{aligned}
\ddot{\boldsymbol{\sigma}}= & \mathbf{H}^{-1}(\boldsymbol{\sigma})(-\mathbf{C}(\boldsymbol{\sigma}, \dot{\boldsymbol{\sigma}}) \dot{\boldsymbol{\sigma}}-\mathbf{d}(\boldsymbol{\sigma}, \dot{\boldsymbol{\sigma}}, \dot{\gamma}, \dot{\boldsymbol{\Omega}}, \ldots)+\mathbf{F}) \\
= & -\mathbf{H}^{-1}(\boldsymbol{\sigma}) \mathbf{C}(\boldsymbol{\sigma}, \dot{\boldsymbol{\sigma}}) \dot{\boldsymbol{\sigma}}-\mathbf{H}^{-1}(\boldsymbol{\sigma}) \mathbf{d}(\boldsymbol{\sigma}, \dot{\boldsymbol{\sigma}}, \dot{\gamma}, \dot{\Omega}, \ldots) \\
& +\mathbf{H}^{-1}(\boldsymbol{\sigma}) \mathbf{F} .
\end{aligned}
$$

If the equivalent disturbance $\mathbf{d}(\bullet)$ can be estimated precisely, then a simplified controller can be designed by adding the estimated disturbance signal to the control input. In the subsequent development, an adaptive fuzzy system is employed to approximate the uncertain and external disturbances included in the spacecraft actuated by VSCMGs to a sufficient degree of accuracy.

\section{Controller Design}

3.1. Fuzzy Adaptive Disturbance Observer. In this subsection, the FADO is presented. To construct a fuzzy system $\widehat{\mathbf{d}}(\bullet$ | $\Theta)$ that accurately approximates $\mathbf{d}(\bullet)$, the FADO system is designed as follows:

$$
\begin{aligned}
\dot{\boldsymbol{\mu}}= & -\mathbf{H}^{-1}(\boldsymbol{\sigma}) \hat{\mathbf{d}}(\bullet \mid \Theta)+\mathbf{H}^{-1}(\boldsymbol{\sigma})(\mathbf{F}-\mathbf{C}(\boldsymbol{\sigma}, \dot{\boldsymbol{\sigma}}) \dot{\boldsymbol{\sigma}}) \\
& +\mathbf{k}_{1}(\dot{\boldsymbol{\sigma}}-\boldsymbol{\mu})
\end{aligned}
$$

where $\mathbf{k}_{1}$ is a positive scalar and the disturbance observation error

$$
\zeta=\dot{\sigma}-\mu
$$

is included [19]. Combining (16) and (18), the error dynamics of the disturbance observer $\zeta$ can be expressed as

$$
\dot{\zeta}=-\mathbf{k}_{1} \zeta-\mathbf{H}^{-1}(\boldsymbol{\sigma})(\mathbf{d}(\bullet \mid \Theta)-\widehat{\mathbf{d}}(\bullet \mid \Theta)) .
$$

$\zeta \rightarrow 0$ implies that the fuzzy system $\widehat{\mathbf{d}}(\bullet \mid \Theta)$ approaches the actual but unknown equivalent disturbance $\mathbf{d}(\bullet)$. The universal approximation property of the fuzzy system states that the unknown disturbance $\mathbf{d}(\bullet)$ can be described by an optimal fuzzy system $\widehat{\mathbf{d}}\left(\bullet \mid \Theta^{*}\right)$ and a reconstruction error $\Delta \mathbf{d}(\bullet)$ :

$$
\begin{aligned}
\mathbf{d}(\bullet) & =\widehat{\mathbf{d}}\left(\bullet \mid \Theta^{*}\right)+\Delta \mathbf{d}(\bullet) \\
\Theta^{*} & =\arg \min (\sup \|\mathbf{d}(\bullet)-\widehat{\mathbf{d}}(\bullet \mid \Theta)\|) .
\end{aligned}
$$

The error of the adaptive tuning parameter is defined as

$$
\widetilde{\Theta}=\Theta^{*}-\Theta \text {. }
$$

The error term $\Delta \mathbf{d}(\bullet)$ can be assumed to be bounded by

$$
\|\Delta \mathbf{d}(\bullet)\| \leq \bar{\Delta} \mathbf{d},
$$

where $\bar{\Delta} \mathbf{d}$ is a positive number. This assumption is reasonable because of the universal approximation theorem. Then, the dynamics of the disturbance observer error $\zeta$ can be rewritten as

$$
\dot{\zeta}=-\mathbf{k}_{1} \zeta-\mathbf{H}^{-1}(\boldsymbol{\sigma}) \widetilde{\Theta} \mathbf{y}(\bullet)+\bar{\Delta} \mathbf{d}
$$

where $\mathbf{y}(\bullet)$ is a fuzzy basis function $(\mathrm{FBF})$, that is, $y^{i}=$ $\prod_{j=1}^{n} \mu_{A_{j}^{i}}\left(z_{j}\right) / \sum_{i=1}^{r}\left(\prod_{j=1}^{n} \mu_{A_{j}^{i}}\left(z_{j}\right)\right)$, and $\mu_{A_{j}^{i}}\left(z_{j}\right)$ is the member function of the fuzzy variable $z_{j}$.

3.2. Controller Design. In this subsection, the output feedback method is employed to design the controller and the corresponding tuning method of the FADO is developed.

Define the control error $\boldsymbol{\sigma}_{e}=\boldsymbol{\sigma}-\boldsymbol{\sigma}_{d}, \dot{\boldsymbol{\sigma}}_{r}=\dot{\boldsymbol{\sigma}}_{d}-\lambda \boldsymbol{\sigma}_{e}$ and the measurement of the attitude tracking error $s=\dot{\boldsymbol{\sigma}}_{e}+\lambda \boldsymbol{\sigma}_{e}=$ $\dot{\boldsymbol{\sigma}}-\dot{\boldsymbol{\sigma}}_{r}$, where $\boldsymbol{\sigma}_{d}$ is the MRP vector presenting the attitude of the desired reference frame with respect to the inertial frame.

Let a control law be such that

$$
\begin{aligned}
\mathbf{F}= & \mathbf{H}(\boldsymbol{\sigma}) \ddot{\boldsymbol{\sigma}}_{r}+\mathbf{C}(\boldsymbol{\sigma}, \dot{\boldsymbol{\sigma}}) \dot{\boldsymbol{\sigma}}_{r} \\
& +\widehat{\mathbf{d}}\left(\boldsymbol{\sigma}, \dot{\boldsymbol{\sigma}}, \boldsymbol{\Omega}, \dot{\boldsymbol{\gamma}}, \dot{\boldsymbol{\Omega}}, \mathbf{s}, \boldsymbol{\sigma}_{d}, \dot{\boldsymbol{\sigma}}_{d}\right)-\mathbf{K}_{p} \mathbf{s} \\
& -\frac{1}{2} \mathbf{G}^{-T}(\boldsymbol{\sigma}) \dot{\mathbf{J G}}^{-1}(\boldsymbol{\sigma}) \mathbf{s},
\end{aligned}
$$

where $\mathbf{K}_{p}$ is a symmetrical positive definite matrix.

One finds the dynamics in terms of $\mathbf{s}$ as follows:

$$
\begin{aligned}
\mathbf{H}(\boldsymbol{\sigma}) \dot{\mathbf{s}}= & -\mathbf{C}(\boldsymbol{\sigma}, \dot{\boldsymbol{\sigma}}) \mathbf{s}-\mathbf{d}(\bullet)+\widehat{\mathbf{d}}(\bullet)-\mathbf{K}_{p} \mathbf{s} \\
& -\frac{1}{2} \mathbf{G}^{-T}(\boldsymbol{\sigma}) \dot{\mathbf{J}} \mathbf{G}^{-1}(\boldsymbol{\sigma}) \mathbf{s} \\
= & -\mathbf{C}(\boldsymbol{\sigma}, \dot{\boldsymbol{\sigma}}) \mathbf{s}+\widetilde{\boldsymbol{\Theta}} \mathbf{y}(z)+\Delta \mathbf{d}-\mathbf{K}_{p} \mathbf{s} \\
& -\frac{1}{2} \mathbf{G}^{-T}(\boldsymbol{\sigma}) \dot{J G}^{-1}(\boldsymbol{\sigma}) \mathbf{s} ;
\end{aligned}
$$

that is,

$$
\begin{aligned}
\dot{\mathbf{s}}= & -\mathbf{H}^{-1}(\boldsymbol{\sigma})\left(\mathbf{C}(\boldsymbol{\sigma}, \dot{\boldsymbol{\sigma}})+\mathbf{K}_{p}+\frac{1}{2} \mathbf{G}^{-T}(\boldsymbol{\sigma}) \dot{\mathbf{J}} \mathbf{G}^{-1}(\boldsymbol{\sigma})\right) \mathbf{s} \\
& +\mathbf{H}^{-1}(\boldsymbol{\sigma}) \widetilde{\Theta} \mathbf{y}(z)+\mathbf{H}^{-1}(\boldsymbol{\sigma}) \Delta \mathbf{d} .
\end{aligned}
$$

By combining (23) and (26), we obtain the following augmented system:

$$
\begin{aligned}
& \left(\begin{array}{c}
\dot{\mathbf{s}} \\
\dot{\zeta}
\end{array}\right) \\
& =\left[\begin{array}{cc}
-\mathbf{H}(\boldsymbol{\sigma})^{-1}\left(\mathbf{C}(\boldsymbol{\sigma}, \dot{\boldsymbol{\sigma}})+\mathbf{K}_{p}+\frac{1}{2} \mathbf{G}^{-T} \dot{\mathbf{J}} \mathbf{G}^{-1}\right) & 0 \\
0 & -\mathbf{k}_{1}
\end{array}\right]\left(\begin{array}{l}
\mathbf{s} \\
\zeta
\end{array}\right) \\
& +\left(\begin{array}{c}
\mathbf{H}^{-1} \\
-\mathbf{H}^{-1}
\end{array}\right) \widetilde{\boldsymbol{\Theta}} \mathbf{y}+\left(\begin{array}{c}
\mathbf{H}^{-1} \\
\mathbf{I}
\end{array}\right) \Delta \mathbf{d} .
\end{aligned}
$$

Or, more compactly,

$$
\dot{\Xi}=\Pi \Xi+\overline{\mathbf{B}} \widetilde{\Theta} \mathbf{y}+\overline{\mathbf{C}} \Delta \mathbf{d},
$$


where

$$
\begin{aligned}
\boldsymbol{\Xi} & =\left(\begin{array}{c}
\dot{\mathbf{s}} \\
\dot{\boldsymbol{\zeta}}
\end{array}\right) \cdot \boldsymbol{\Pi} \\
& =\left[\begin{array}{cc}
-\mathbf{H}(\boldsymbol{\sigma})^{-1}\left(\mathbf{C}(\boldsymbol{\sigma}, \dot{\boldsymbol{\sigma}})+\mathbf{K}_{p}+\frac{1}{2} \mathbf{G}^{-T} \mathbf{J G}^{-1}\right) & 0 \\
0 & -\mathbf{k}_{1}
\end{array}\right], \\
\overline{\mathbf{B}} & =\left(\begin{array}{c}
\mathbf{H}^{-1} \\
-\mathbf{H}^{-1}
\end{array}\right), \\
\overline{\mathbf{C}} & =\left(\begin{array}{c}
\mathbf{H}^{-1} \\
\mathbf{I}
\end{array}\right) .
\end{aligned}
$$

Here, the control error and fuzzy approximation error used in the system are referred to as the total error $\boldsymbol{\Xi}$ in the remainder of this paper. In addition, as explained below, the system matrix $\Pi$ can be designed such that all of the eigenvalues are in the open left half plane by the judicious choice of the controller gains $\left(\mathbf{K}_{p}\right)$ and the gains of the fuzzy approximation error estimation $\left(\mathbf{k}_{1}\right)$. Then, there exists a symmetric positive definite matrix $\mathbf{P}$ such that

$$
\Pi^{T} \mathbf{P}+\mathbf{P} \Pi=-\mathbf{Q} .
$$

For an arbitrary positive definite matrix $\mathbf{Q}$, let the Lyapunov function candidate for subsystem (30) be given by

$$
V_{1}=\frac{1}{2 \lambda_{\max }(\mathbf{P})} \boldsymbol{\Xi}^{T} \mathbf{P} \boldsymbol{\Xi}+\frac{1}{2} \operatorname{tr}\left(\widetilde{\boldsymbol{\Theta}}^{T} \boldsymbol{\Lambda} \widetilde{\boldsymbol{\Theta}}\right) .
$$

Differentiating (29) and using the skew-symmetry of the matrix $\left(\dot{\mathbf{H}}(\boldsymbol{\sigma})-2 \mathbf{C}(\boldsymbol{\sigma}, \dot{\boldsymbol{\sigma}})-\mathbf{G}^{-T} \dot{J G}^{-1}\right)$, one obtains

$$
\begin{aligned}
& \dot{V}_{1}=\frac{1}{\lambda_{\max }(\mathbf{P})} \mathbf{\Xi}^{T} \mathbf{P} \dot{\boldsymbol{\Xi}}+\operatorname{tr}\left(\widetilde{\boldsymbol{\Theta}}^{T} \boldsymbol{\Lambda} \dot{\widetilde{\boldsymbol{\Theta}}}\right) \\
& =\frac{1}{\lambda_{\max }(\mathbf{P})}\left(\boldsymbol{\Xi}^{T} \mathbf{P} \boldsymbol{\Pi} \boldsymbol{\Xi}+\boldsymbol{\Xi}^{T} \mathbf{P} \overline{\mathbf{B}} \widetilde{\Theta} \mathbf{y}+\boldsymbol{\Xi}^{T} \mathbf{P} \overline{\mathbf{C}} \Delta \mathbf{d}\right) \\
& +\operatorname{tr}\left(\widetilde{\boldsymbol{\Theta}}^{T} \boldsymbol{\Lambda} \dot{\widetilde{\boldsymbol{\Theta}}}\right)=\frac{1}{\lambda_{\max }(\mathbf{P})}\left(\frac{1}{2} \boldsymbol{\Xi}^{T}\left(\mathbf{P} \boldsymbol{\Pi}+\boldsymbol{\Pi}^{T} \mathbf{P}\right) \boldsymbol{\Xi}\right. \\
& \left.+\operatorname{tr}\left\{\mathbf{y}^{T} \widetilde{\boldsymbol{\Theta}}^{T} \overline{\mathbf{B}}^{T} \mathbf{P} \boldsymbol{\Xi}\right\}+\boldsymbol{\Xi}^{T} \mathbf{P} \overline{\mathbf{C}} \Delta \mathbf{d}\right)+\operatorname{tr}\left(\widetilde{\boldsymbol{\Theta}}^{T} \boldsymbol{\Lambda} \dot{\widetilde{\boldsymbol{\Theta}}}\right) \\
& =-\frac{1}{2 \lambda_{\max }(\mathbf{P})} \boldsymbol{\Xi}^{T} \mathbf{Q} \boldsymbol{\Xi}+\frac{1}{\lambda_{\max }(\mathbf{P})} \boldsymbol{\Xi}^{T} \mathbf{P} \overline{\mathbf{C}} \Delta \mathbf{d} \\
& +\operatorname{tr}\left\{\widetilde{\boldsymbol{\Theta}}^{T}\left(\boldsymbol{\Lambda} \dot{\widetilde{\mathbf{\Theta}}}+\frac{1}{\lambda_{\max }(\mathbf{P})} \overline{\mathbf{B}}^{T} \mathbf{P} \boldsymbol{\Xi} \mathbf{y}^{T}\right)\right\} .
\end{aligned}
$$

By choosing the fuzzy rule tuning method to be

$$
\dot{\widetilde{\boldsymbol{\Theta}}}=-\frac{1}{\lambda_{\max }(\mathbf{P})} \boldsymbol{\Lambda}^{-1} \overline{\mathbf{B}}^{T} \mathbf{P} \boldsymbol{\Xi} \mathbf{y}^{T}
$$

or, equivalently,

$$
\dot{\boldsymbol{\Theta}}=\frac{1}{\lambda_{\max }(\mathbf{P})} \boldsymbol{\Lambda}^{-1} \overline{\mathbf{B}}^{T} \mathbf{P} \boldsymbol{\Xi} \mathbf{y}^{T}
$$

one obtains

$$
\begin{aligned}
& \dot{V}_{1}=-\frac{1}{2 \lambda_{\max }(\mathbf{P})} \boldsymbol{\Xi}^{T} \mathbf{Q} \boldsymbol{\Xi}+\frac{1}{\lambda_{\max }(\mathbf{P})} \boldsymbol{\Xi}^{T} \mathbf{P} \overline{\mathbf{C}} \Delta \mathbf{d} \leq-\frac{1}{2} \\
& \cdot \frac{\lambda_{\min }(\mathbf{Q})}{\lambda_{\max }(\mathbf{P})}\|\boldsymbol{\Xi}\|^{2}+\frac{1}{\lambda_{\max }(\mathbf{P})} \boldsymbol{\Xi}^{T} \mathbf{P} \overline{\mathbf{C}} \Delta \mathbf{d}=-\frac{1}{2} \\
& \cdot \frac{\lambda_{\min }(\mathbf{Q})}{\lambda_{\max }(\mathbf{P})}\|\boldsymbol{\Xi}\|^{2} \\
& -\left(\frac{1}{2} \frac{\sqrt{\lambda_{\min }(\mathbf{Q})}}{\lambda_{\max }(\mathbf{P})} \boldsymbol{\Xi}-\frac{1}{\sqrt{\lambda_{\min }(\mathbf{Q})}} \overline{\mathbf{C}} \Delta \mathbf{d}\right)^{T} \\
& \cdot \mathbf{P}\left(\frac{1}{2} \frac{\sqrt{\lambda_{\min }(\mathbf{Q})}}{\lambda_{\max }(\mathbf{P})} \boldsymbol{\Xi}-\frac{1}{\sqrt{\lambda_{\min }(\mathbf{Q})}} \overline{\mathbf{C}} \Delta \mathbf{d}\right) \leq-\frac{1}{4} \\
& \cdot \frac{\lambda_{\min }(\mathbf{Q})}{\lambda_{\max }(\mathbf{P})}\|\boldsymbol{\Xi}\|^{2}+\frac{\lambda_{\max }(\mathbf{P})}{\lambda_{\min }(\mathbf{Q})}\|\overline{\mathbf{C}} \Delta \mathbf{d}\|^{2}=-\frac{1}{2} \\
& \cdot \frac{\lambda_{\text {min }}(\mathbf{Q})}{2 \lambda_{\max }(\mathbf{P})}\|\boldsymbol{\Xi}\|^{2}+\frac{1}{2} \frac{2 \lambda_{\max }(\mathbf{P})}{\lambda_{\min }(\mathbf{Q})}\|\overline{\mathbf{C}} \Delta \mathbf{d}\|^{2}
\end{aligned}
$$

and $\dot{V}_{1}$ is negative definite outside the compact set $\mathbf{B}_{\Xi}$

$$
\mathbf{B}_{\Xi}=\left\{\boldsymbol{\Xi} \mid\|\boldsymbol{\Xi}\| \leq \frac{2 \lambda_{\max }(\mathbf{P})}{\lambda_{\min }(\mathbf{Q})}\|\overline{\mathbf{C}} \Delta \mathbf{d}\|\right\} .
$$

Then, assuming that $\boldsymbol{\Theta}$ is bounded, the tracking error $\boldsymbol{\Xi}$ is globally uniformly ultimately bounded (UUB) outside the set $\mathbf{B}_{\Xi}$.

3.3. Solution of the Velocity Steering Law for IPACS. From (24), it follows that the required control inputs are obtained by solving [4]

$$
\begin{aligned}
\mathbf{F}= & \mathbf{G}^{-T}(\boldsymbol{\sigma})\left[\mathbf{h}_{2}^{\times}\right] \boldsymbol{\omega}-\mathbf{G}^{-T}(\boldsymbol{\sigma}) \mathbf{C t}_{c} \\
& -\frac{1}{2} \mathbf{G}^{-T}(\boldsymbol{\sigma}) \dot{\mathbf{J}} \boldsymbol{\omega},
\end{aligned}
$$

$\left[\begin{array}{ll}\mathrm{C} & \mathrm{D}\end{array}\right]\left\{\begin{array}{c}\dot{\gamma} \\ \dot{\boldsymbol{\Omega}}\end{array}\right\}=\mathrm{Ct}_{c}$,

where $\dot{\mathbf{J}}=\mathbf{A}_{t}[\dot{\boldsymbol{\gamma}}]^{d}\left(\mathbf{I}_{c s}-\mathbf{I}_{c t}\right) \mathbf{A}_{s}^{T}+\mathbf{A}_{s}[\dot{\boldsymbol{\gamma}}]^{d}\left(\mathbf{I}_{c s}-\mathbf{I}_{c t}\right) \mathbf{A}_{t}^{T}$,

$$
\begin{aligned}
\mathbf{D} & =\mathbf{A}_{s} \mathbf{I}_{w s} \\
\mathbf{C} & =\mathbf{A}_{t} \mathbf{I}_{w s}[\mathbf{\Omega}]^{d}+\left[\boldsymbol{\omega}^{\times}\right] \mathbf{A}_{g} \mathbf{I}_{c g}+\frac{1}{2}\left[\left(e_{s 1} e_{t 1}^{T}+e_{t 1} e_{s 1}^{T}\right)\right. \\
& \left.\cdot\left(\boldsymbol{\omega}+\mathbf{G}^{-1} \dot{\boldsymbol{\sigma}}_{r}\right), \ldots,\left(e_{s N} e_{t N}^{T}+e_{t N} e_{s N}^{T}\right)\left(\boldsymbol{\omega}+\mathbf{G}^{-1} \dot{\boldsymbol{\sigma}}_{r}\right)\right] \\
& \cdot\left(\mathbf{I}_{c s}-\mathbf{I}_{c t}\right)
\end{aligned}
$$

$$
\begin{aligned}
\mathbf{C t}_{c} & =-\mathbf{G}^{T}(\boldsymbol{\sigma})\left(\mathbf{H}(\boldsymbol{\sigma}) \ddot{\boldsymbol{\sigma}}_{r}+\mathbf{C}(\boldsymbol{\sigma}, \dot{\boldsymbol{\sigma}}) \dot{\boldsymbol{\sigma}}_{r}\right. \\
& \left.+\widehat{\mathbf{d}}\left(\boldsymbol{\sigma}, \dot{\boldsymbol{\sigma}}, \boldsymbol{\Omega}, \dot{\boldsymbol{\gamma}}, \dot{\boldsymbol{\Omega}}, \mathbf{s}, \boldsymbol{\sigma}_{d}, \dot{\boldsymbol{\sigma}}_{d}\right)-\mathbf{K}_{p} \mathbf{s}\right)+\left[\mathbf{h}_{2}^{\times}\right] \boldsymbol{\omega} .
\end{aligned}
$$


Once $\dot{\gamma}$ and $\dot{\boldsymbol{\Omega}}$ are known from the solution of (37), they can be substituted into the FADO and control law.

Define

$$
\begin{aligned}
\mathbf{C}_{s} & =\left[\begin{array}{llll}
\frac{\mathbf{C}(1)}{\mathbf{I}_{w s} \Omega_{1}} & \frac{\mathbf{C}(2)}{\mathbf{I}_{w s} \Omega_{2}} & \cdots & \frac{\mathbf{C}(n)}{\mathbf{I}_{w s} \Omega_{n}}
\end{array}\right] \\
\Gamma & =\operatorname{det}\left(\mathbf{C}_{s} \mathbf{C}_{s}^{T}\right),
\end{aligned}
$$

where $\mathbf{C}(i)$ represents the $i$ th column of the matrix $\mathbf{C}$ and $\Gamma$ is a measurement of the CMG singularity. According to $\Gamma$, the VSCMG can operate either as an MW (close to a CMG singularity, i.e., when $\Gamma$ is zero) or as a regular CMG (away from the singularity, i.e., when $\Gamma$ is large). In this work, a scalar $\alpha$ is defined as the measurement of the singularity of the VSCMG cluster:

$$
\alpha= \begin{cases}0 & \text { if } \Gamma \geq \Gamma_{0} \\ \alpha_{0}\left(\Gamma-\Gamma_{0}\right)^{2} & \text { if } \Gamma<\Gamma_{0},\end{cases}
$$

where $\alpha_{0}$ and $\Gamma_{0}$ are positive scalar parameters. When $\Gamma$ is no less than $\Gamma_{0}, \alpha=0$ indicates that the CMG is not close to singularity; otherwise, the CMG is close to singularity.

(1) The Case of $\alpha \neq 0$. The solution of the gimbal rates is based on a robust pseudo inverse steering law:

$$
\dot{\gamma}=\mathbf{C}^{T}\left(\mathbf{C C}^{T}+\alpha \mathbf{I}\right)^{-1} \mathbf{C t}_{c}
$$

where the error torque $\Delta \mathbf{C t}$ between the output torque of the CMG and the commanded torque $\mathbf{C t}_{c}$ is

$$
\Delta \mathbf{C t}=\left(\mathbf{I}-\mathbf{C C}^{T}\left(\mathbf{C} \mathbf{C}^{T}+\alpha \mathbf{I}\right)^{-1}\right) \mathbf{C t}_{c} .
$$

The error torque can be generated by MW mode; that is,

$$
\mathrm{D} \dot{\mathbf{\Omega}}=\Delta \mathbf{C t}_{c}
$$

at the same time, the gyro rotors should realize the power tracking; that is,

$$
\mathbf{\Omega}^{T} \mathbf{I}_{w s} \dot{\mathbf{\Omega}}=P(t) .
$$

Then, the solution of (43) is

$$
\dot{\mathbf{\Omega}}=\mathbf{D}^{T}\left(\mathbf{D D}^{T}\right)^{-1} \Delta \mathbf{C t}+\dot{\mathbf{\Omega}}_{n}
$$

where $\dot{\boldsymbol{\Omega}}_{n}$ is the null motion, which does not affect the generated output torque but satisfies (44):

$$
\begin{aligned}
& \dot{\boldsymbol{\Omega}}_{n}=\mathbf{S}\left(\boldsymbol{\Omega}^{T} \mathbf{I}_{w s}\right)^{T}\left(\boldsymbol{\Omega}^{T} \mathbf{I}_{w s} \mathbf{S}\left(\boldsymbol{\Omega}^{T} \mathbf{I}_{w s}\right)^{T}\right)^{-1} P_{m}, \\
& \mathbf{S}=\mathbf{I}-\mathbf{D}^{T}\left(\mathbf{D D}^{T}\right)^{-1} \mathbf{D}, P_{m}=P(t)-\left(\mathbf{I}_{w s} \boldsymbol{\Omega}^{T}\right) \mathbf{D}^{T}\left(\mathbf{D D}^{T}\right)^{-1} \Delta \mathbf{C t} .
\end{aligned}
$$

(2) The Case of $\alpha=0$. In this situation, the CMG is away from singularity, and the gyro rotors realize power tracking. Then, the weighted pseudo inverse solution of (44) is

$$
\dot{\boldsymbol{\Omega}}=\mathbf{W}\left(\boldsymbol{\Omega}^{T} \mathbf{I}_{w s}\right)^{T}\left(\boldsymbol{\Omega}^{T} \mathbf{I}_{w s} \mathbf{W}\left(\boldsymbol{\Omega}^{T} \mathbf{I}_{w s}\right)^{T}\right)^{-1} P(t),
$$

where $\mathbf{W}=\operatorname{diag}\left(W_{1}, W_{2}, \ldots, W_{n}\right)$,

$$
W_{i}=\exp \left(\beta_{0} \operatorname{sign}(P(t))\left(1-\frac{\Omega_{i}}{\Omega_{b i}}\right)\right) .
$$

$\beta_{0}$ is the positive parameter, and $\Omega_{b i}$ is the average velocity of the rotors:

$$
\Omega_{b i}=\sqrt{\left(\frac{2}{n \mathbf{I}_{w s}} \int_{t_{0}}^{t} P(t) d t+\frac{1}{n} \boldsymbol{\Omega}_{0}^{T} \boldsymbol{\Omega}_{0}\right)} .
$$

The output torque of the gyro rotors is

$$
\mathbf{C} \mathbf{t}_{\mathbf{r}}=\mathbf{D W}\left(\boldsymbol{\Omega}^{T} \mathbf{I}_{w s}\right)^{T}\left(\boldsymbol{\Omega}^{T} \mathbf{I}_{w s} W\left(\boldsymbol{\Omega}^{T} \mathbf{I}_{w s}\right)^{T}\right)^{-1} P(t) .
$$

Then, the solution of the robust pseudo inverse steering law is

$$
\dot{\gamma}=\mathbf{C}^{T}\left(\mathbf{C C}^{T}+\alpha \mathbf{I}\right)^{-1}\left(\mathbf{C} \mathbf{t}_{c}-\mathbf{C t}_{r}\right)
$$

\section{Simulation Study}

To validate the proposed scheme, a numerical example of a satellite in a maneuvering motion with an unknown mass moment of inertia and external disturbances is presented. The satellite is actuated by a standard four-VSCMG pyramid configuration, and the initial MRPs are set to

$$
\boldsymbol{\sigma}_{d 0}=\left\{\begin{array}{lll}
0 & 0 & 0
\end{array}\right\}^{T} .
$$

The desired attitude trajectory is selected to be a smooth rotation along each axis with the following corresponding desired angular velocity:

$$
\begin{aligned}
& \boldsymbol{\omega}_{d}(t) \\
& =0.02\left\{\sin \left(\frac{2 \pi t}{800}\right) \sin \left(\frac{2 \pi t}{600}\right) \sin \left(\frac{2 \pi t}{400}\right)\right\}^{T} \mathrm{rad} / \mathrm{s} .
\end{aligned}
$$

The corresponding desired MRPs are shown in Figure 1, and the power tracking profile is shown in Figure 2. Table 1 provides the nominal parameters and initial conditions of the spacecraft, and Table 2 provides the actual mass moment of the inertia of the spacecraft and the actuator.

The external disturbances in (12) are

$$
\mathbf{g}_{e}=\left\{0.05+0.25 \sin \left(\frac{2 \pi t}{200}\right) 0.05+0.25 \sin \left(\frac{2 \pi t}{150}\right) 0.05+0.25 \sin \left(\frac{2 \pi t}{100}\right)\right\}^{T} \mathrm{rad} / \mathrm{s}
$$


TABLE 1: Nominal parameters and initial conditions of the simulation.

\begin{tabular}{|c|c|c|}
\hline Symbol & Value & Unit \\
\hline$N$ & 4 & \\
\hline$\theta$ & 54.75 & deg \\
\hline $\boldsymbol{\omega}_{0}$ & $\left\{\begin{array}{lll}0 & 0 & 0\end{array}\right\}^{T}$ & $\mathrm{rad} / \mathrm{s}$ \\
\hline$\dot{\boldsymbol{\omega}}_{0}$ & $\left\{\begin{array}{lll}0 & 0 & 0\end{array}\right\}^{T}$ & $\mathrm{rad} / \mathrm{s}^{2}$ \\
\hline$\sigma_{0}$ & $\left\{\begin{array}{lll}0 & 0 & 0\end{array}\right\}^{T}$ & \\
\hline$\gamma_{0}$ & $\{\pi / 2-\pi / 2-\pi / 2 \pi / 2\}^{T}$ & $\mathrm{rad}$ \\
\hline$\dot{\gamma}_{0}$ & $\left\{\begin{array}{llll}0 & 0 & 0 & 0\end{array}\right\}^{T}$ & $\mathrm{rad} / \mathrm{s}$ \\
\hline $\mathbf{\Omega}_{0}$ & $1000\left\{\begin{array}{llll}1 & 1 & 1 & 1\end{array}\right\}^{T}$ & $\mathrm{rad} / \mathrm{s}$ \\
\hline$\dot{\mathbf{\Omega}}_{0}$ & $\left\{\begin{array}{llll}0 & 0 & 0 & 0\end{array}\right\}^{T}$ & $\mathrm{rad} / \mathrm{s}^{2}$ \\
\hline $\mathbf{J}_{0}$ & {$\left[\begin{array}{rrr}13000 & 2400 & -1200 \\
2400 & 5000 & 2400 \\
-1200 & 2400 & 9000\end{array}\right]$} & $\mathrm{kg} \cdot \mathrm{m}^{2}$ \\
\hline $\mathbf{I}_{w s}$ & $\operatorname{diag}\left(\begin{array}{llll}0.84 & 0.84 & 0.84 & 0.84\end{array}\right)$ & $\mathrm{kg} \cdot \mathrm{m}^{2}$ \\
\hline $\mathbf{I}_{w t}, \mathbf{I}_{w g}$ & $\operatorname{diag}\left(\begin{array}{llll}0.48 & 0.48 & 0.48 & 0.48\end{array}\right)$ & $\mathrm{kg} \cdot \mathrm{m}^{2}$ \\
\hline $\mathbf{I}_{g t}, \mathbf{I}_{g g}$ & $\operatorname{diag}\left(\begin{array}{llll}0.12 & 0.12 & 0.12 & 0.12\end{array}\right)$ & $\mathrm{kg} \cdot \mathrm{m}^{2}$ \\
\hline
\end{tabular}

TABLE 2: Actual parameters.

\begin{tabular}{lcc}
\hline Symbol & Value & Unit \\
\hline$\breve{\mathbf{J}}_{0}$ & {$\left[\begin{array}{rrrr}15053 & 3000 & -1000 \\
3000 & 6510 & 2000 \\
-1000 & 2000 & 11122\end{array}\right]$} & $\mathrm{kg} \cdot \mathrm{m}^{2}$ \\
$\breve{\mathbf{I}}_{w s}$ & $\operatorname{diag}\left(\begin{array}{llll}0.7 & 0.7 & 0.7 & 0.7\end{array}\right)$ & $\mathrm{kg} \cdot \mathrm{m}^{2}$ \\
$\breve{\mathbf{I}}_{w t}, \breve{\mathbf{I}}_{w g}$ & $\operatorname{diag}\left(\begin{array}{llll}0.4 & 0.4 & 0.4 & 0.4\end{array}\right)$ & $\mathrm{kg} \cdot \mathrm{m}^{2}$ \\
$\breve{\mathbf{I}}_{g t}, \breve{\mathbf{I}}_{g g}$ & $\operatorname{diag}\left(\begin{array}{llll}0.1 & 0.1 & 0.1 & 0.1\end{array}\right)$ & $\mathrm{kg} \cdot \mathrm{m}^{2}$ \\
\hline
\end{tabular}

The friction matrices $\mathbf{F}_{d}$ and $\mathbf{F}_{s}$ in (10) are

$$
\begin{aligned}
& \mathbf{F}_{d}=0.002 \mathbf{I}_{3 \times 4}, \\
& \mathbf{F}_{s}=0.004 \mathbf{I}_{3 \times 4},
\end{aligned}
$$

where

$$
\mathbf{I}_{3 \times 4}=\left[\begin{array}{llll}
1 & 0 & 0 & 0 \\
0 & 1 & 0 & 0 \\
0 & 0 & 1 & 0
\end{array}\right] \text {. }
$$

In addition, the initial errors of the direction cosine matrices of the VSCMGs are as follows:

$$
\begin{aligned}
\breve{\mathbf{A}}_{s 0} & =\left[\begin{array}{cccc}
-0.8160 & -0.0004 & -0.8160 & 0.0002 \\
-0.0002 & 0.8160 & -0.0003 & 0.8160 \\
0.5770 & -0.5770 & -0.5770 & 0.5770
\end{array}\right], \\
\breve{\mathbf{A}}_{t 0} & =\left[\begin{array}{cccc}
-0.0003 & -1.0003 & 0.0002 & -1.0003 \\
-1.0002 & -0.0005 & -1.0005 & 0.0004 \\
0.0001 & 0.0002 & 0.0004 & 0.0003
\end{array}\right] .
\end{aligned}
$$
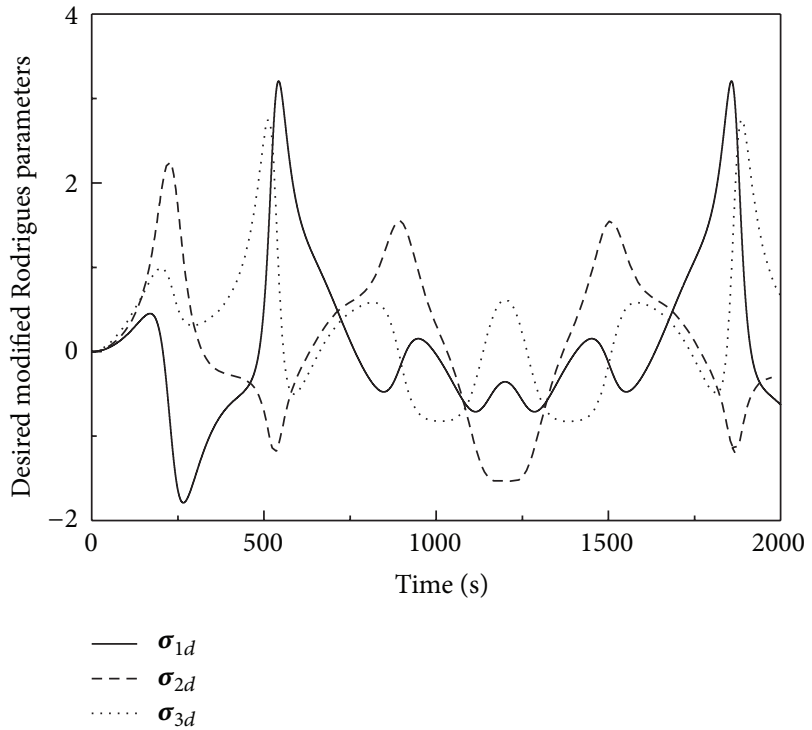

Figure 1: Desired $\sigma_{d}$ profile.

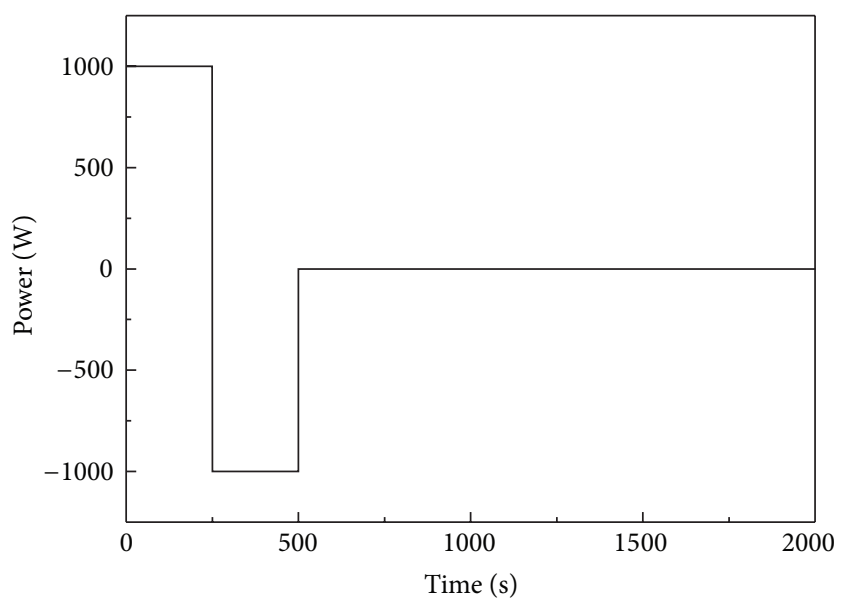

Figure 2: Desired power profile.

Table 3 provides the parameters used in the numerical simulation.

For the fuzzy system in the designed FADO, the membership functions for each variable are selected as follows:

$$
\begin{aligned}
& \mu_{A^{1}(x)}=\frac{1}{1+e^{(5(x+0.8 \times a))},} \\
& \mu_{A^{2}(x)}=e^{-((x+0.6 \times a) / w)^{2}}, \\
& \mu_{A^{3}(x)}=e^{-((x+0.4 \times a) / w)^{2}}, \\
& \mu_{A^{4}(x)}=e^{-((x+0.2 \times a) / w)^{2}}, \\
& \mu_{A^{5}(x)}=e^{-(x / w)^{2}}, \\
& \mu_{A^{6}(x)}=e^{-((x-0.2 \times a) / w)^{2}},
\end{aligned}
$$




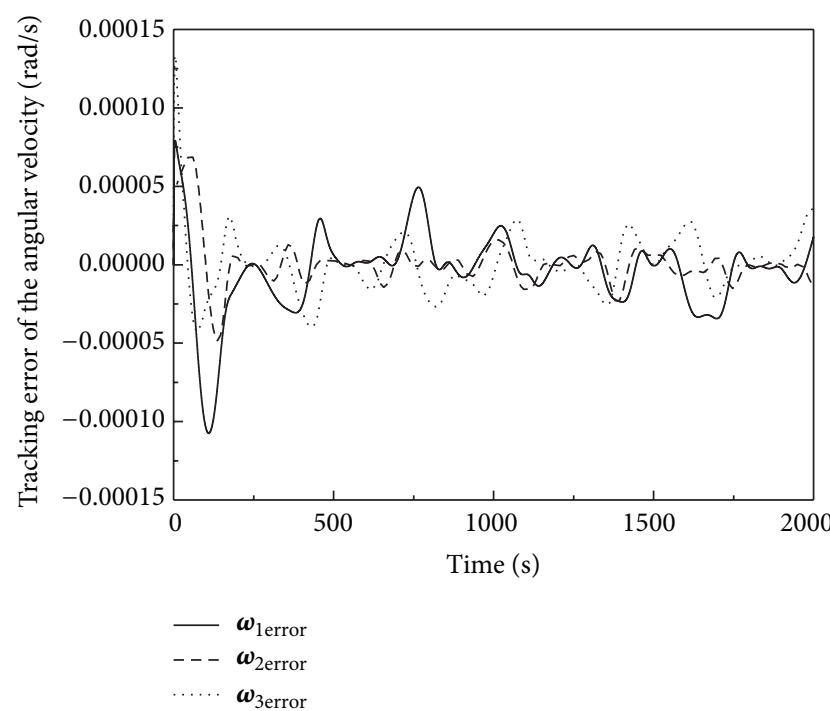

(a) With FADO

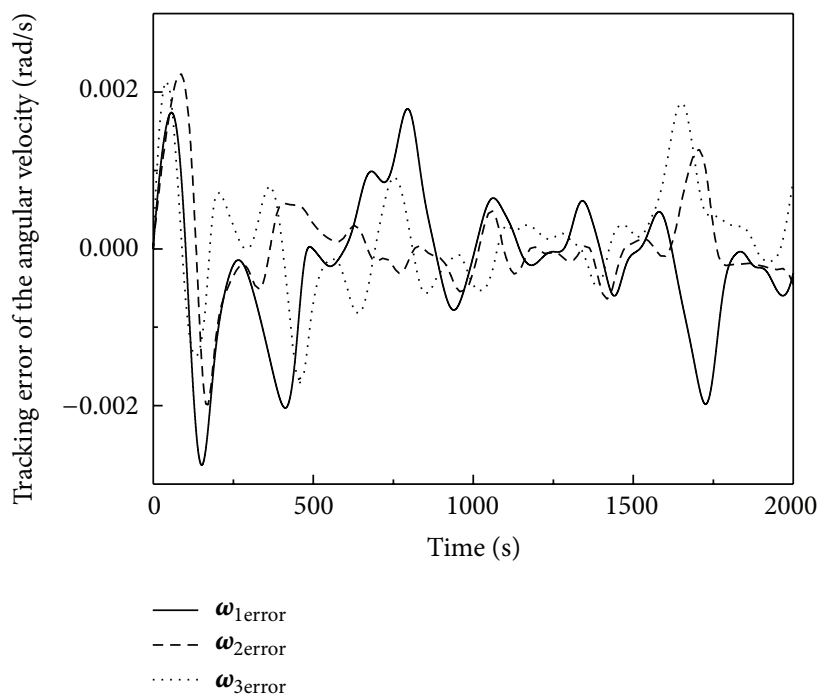

(b) Without a FADO

FIgURE 3: Comparison of the tracking errors of the angular velocity.

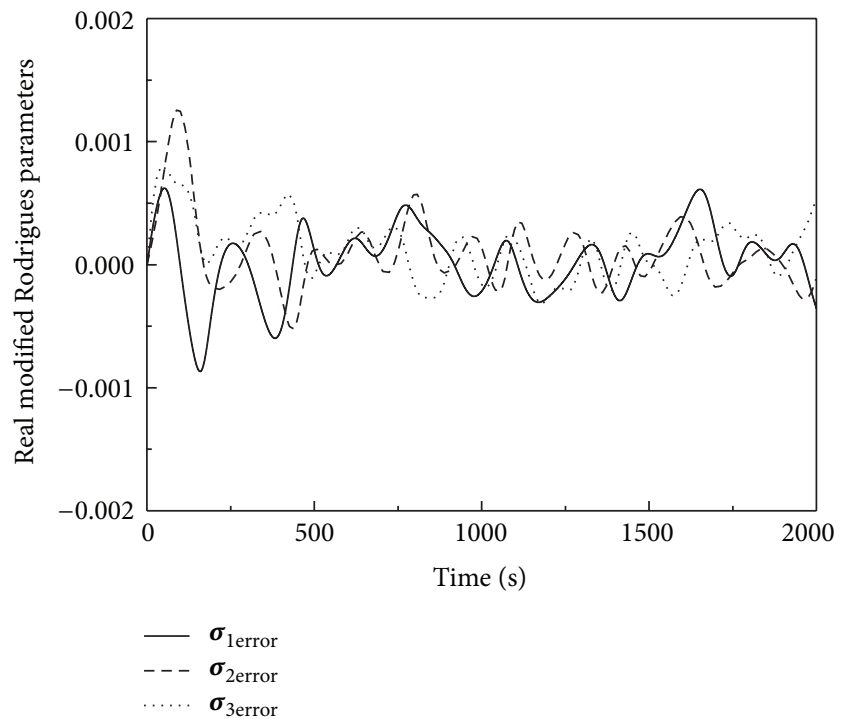

(a) With FADO

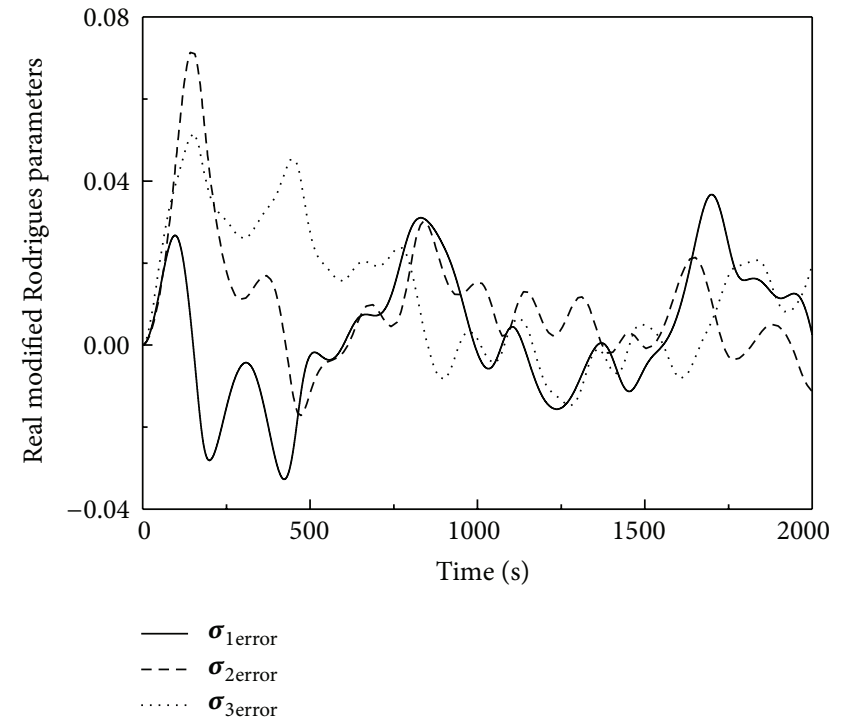

(b) Without a FADO

FIGURE 4: Comparison of the MRP errors.

$$
\begin{aligned}
& \mu_{A^{7}(x)}=e^{-((x-0.4 \times a) / w)^{2}}, \\
& \mu_{A^{8}(x)}=e^{-((x-0.6 \times a) / w)^{2}}, \\
& \mu_{A^{9}(x)}=\frac{1}{1+e^{(-5(x-0.8 \times a))}} .
\end{aligned}
$$

Table 4 provides the values of $a$ and $w$ for each variable. In this simulation, the adaptive fuzzy system $\widehat{\mathbf{d}}(\bullet)$ uses the rule base with fuzzy rules of the following form:

$$
\text { if } x \text { is } A_{i} \text {, then } \widehat{\mathbf{d}}(\bullet) \text { is } B_{i} \quad(i=1, \ldots, 9) \text {, }
$$

where the premise variable $x$ is one of the variables provided in Table 1; that is, we use only one variable for each rule to achieve an optimal balance between the interpretability and accuracy by reducing the number of fuzzy rules and the number of input variables [20]. The adaptive fuzzy system $\widehat{\mathbf{d}}(\bullet)$ uses $\boldsymbol{\sigma}, \dot{\boldsymbol{\sigma}}, \boldsymbol{\Omega}, \dot{\boldsymbol{\gamma}}$, and $\dot{\boldsymbol{\Omega}}$ as input arguments, and the rule base is composed of $162(9 \times 18)$ fuzzy rules. The adaptive fuzzy logic scheme has a simple structure and high computational efficiency because no physical parameters need to be estimated online; thus, the proposed scheme is more suitable for real-time control applications.

Figure 3 presents the tracking error of the angular velocity with and without a FADO, and Figure 4 presents the tracking 


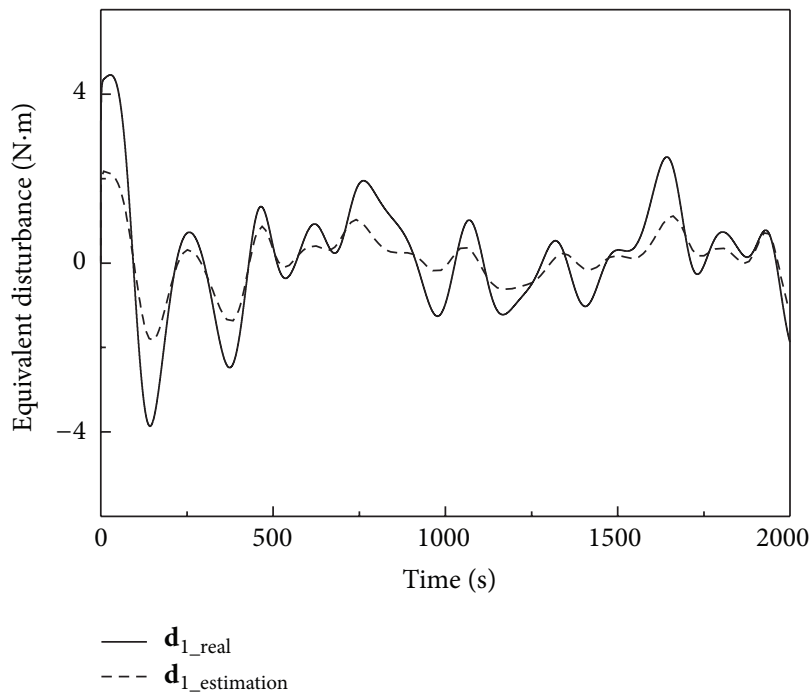

(a)

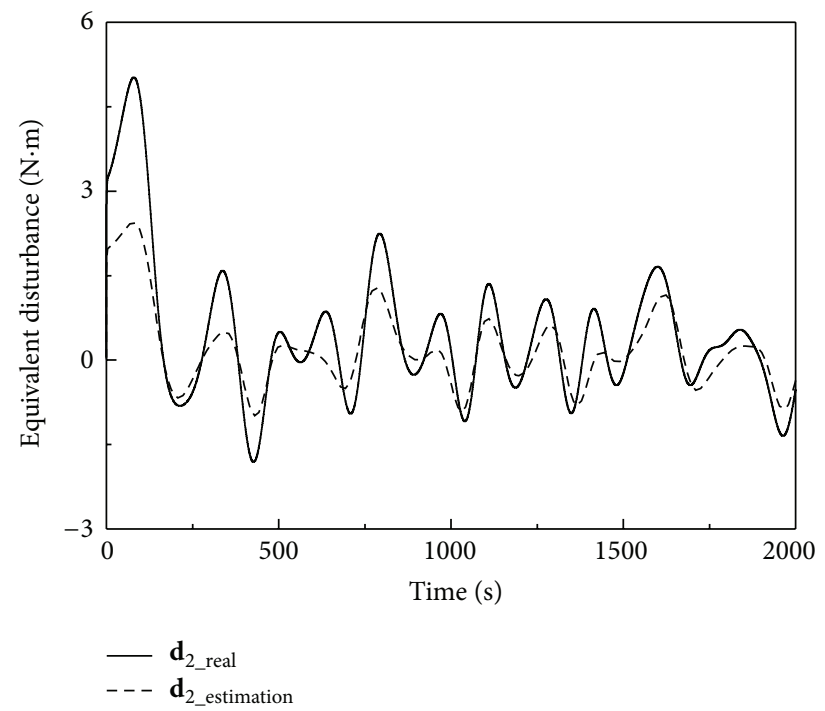

(b)

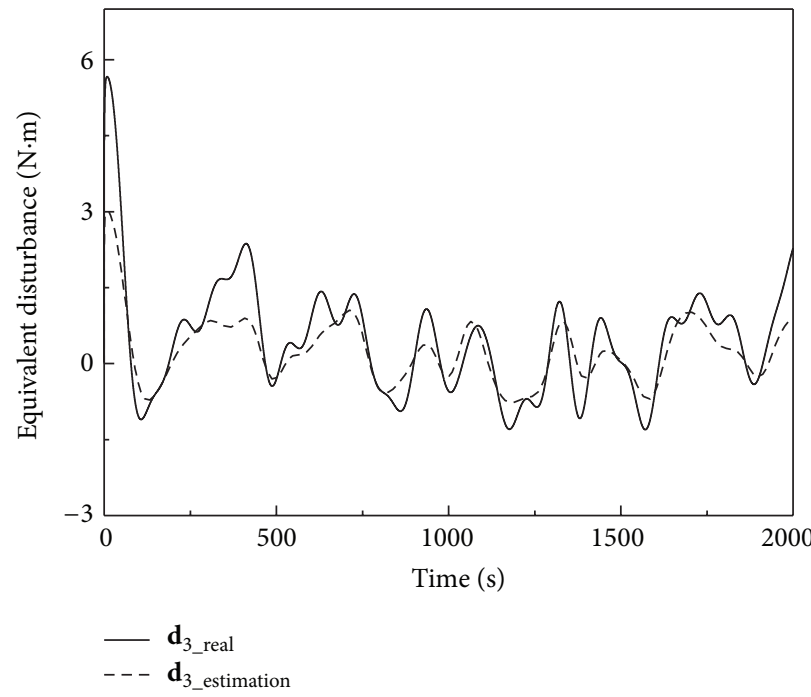

(c)

FIGURE 5: Comparison of the real disturbances and FADO approximation.

TABLE 3: Numerical simulation parameters.

\begin{tabular}{|c|c|}
\hline Symbol & Value \\
\hline $\mathbf{K}_{p}$ & $\operatorname{diag}(4000 ; 4000 ; 4000)$ \\
\hline $\mathbf{k}_{1}$ & 25 \\
\hline $\boldsymbol{\omega}_{0}$ & $\operatorname{diag}(1000 ; 1000 ; 1000)$ \\
\hline$\lambda$ & 1 \\
\hline$\alpha_{0}$ & 3750 \\
\hline$\Gamma_{0}$ & 0.2 \\
\hline$\beta_{0}$ & 50 \\
\hline$\dot{\Omega}_{0}$ & $\left\{\begin{array}{llll}0 & 0 & 0 & 0\end{array}\right\}^{T}$ \\
\hline
\end{tabular}

error of the MRPs. The simulation results illustrate that the spacecraft attitude tracks the desired attitude trajectory effectively after a short period of time, and the proposed controller
TABLE 4: Premise parameters for each variable used in the FADO.

\begin{tabular}{lcc}
\hline Var $x$ & $a$ & $w$ \\
\hline $\boldsymbol{\sigma}$ & 4 & 4 \\
$\dot{\boldsymbol{\sigma}}$ & 0.1 & 0.1 \\
$\boldsymbol{\Omega}$ & 14,000 & 14,000 \\
$\dot{\boldsymbol{\Omega}}$ & 2 & 2 \\
$\dot{\boldsymbol{\gamma}}$ & 0.005 & 0.005 \\
\hline
\end{tabular}

outperforms the controller without a FADO. Figure 5 presents the unknown functions approximated by the FADO, and Figure 6 compares the control torques for the controllers with and without a FADO. Finally, Figures $7-10$ provide the gimbal angles and wheel speed histories of the two controllers. From the simulation, it can be seen that the presented FADO 


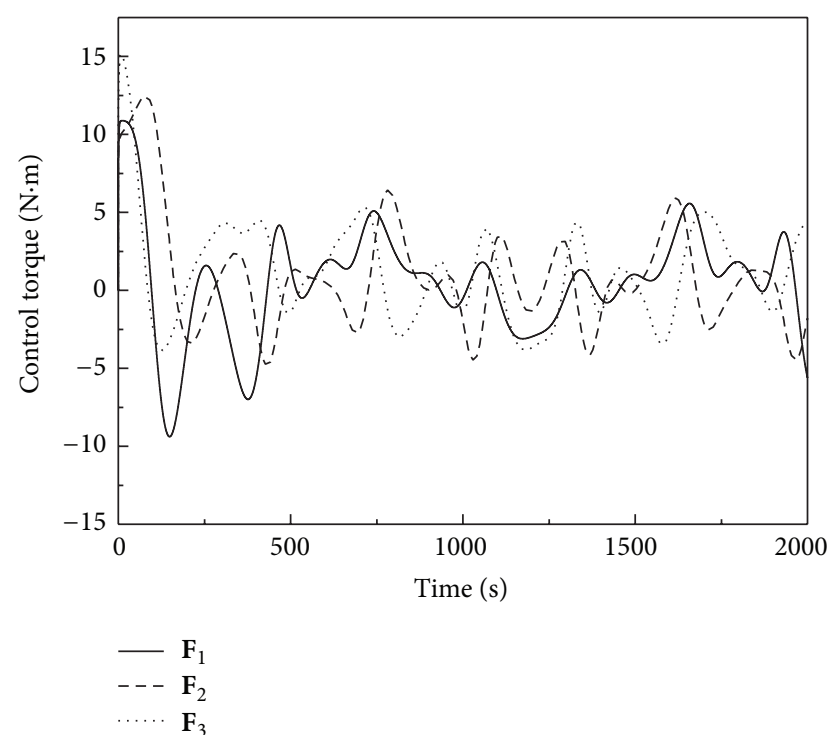

(a) With FADO

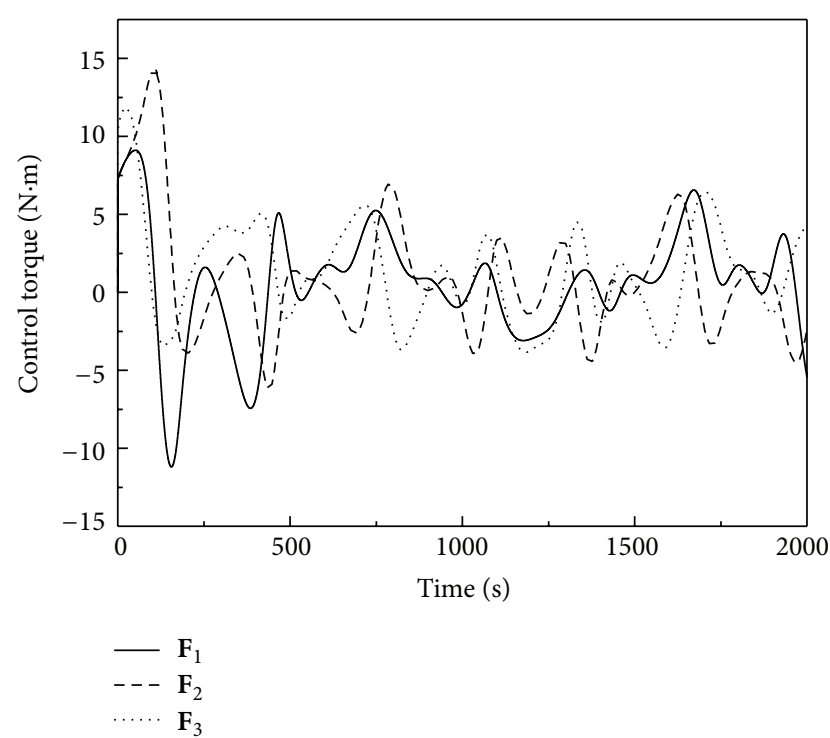

(b) Without a FADO

FIGURE 6: Comparison of the control torques.

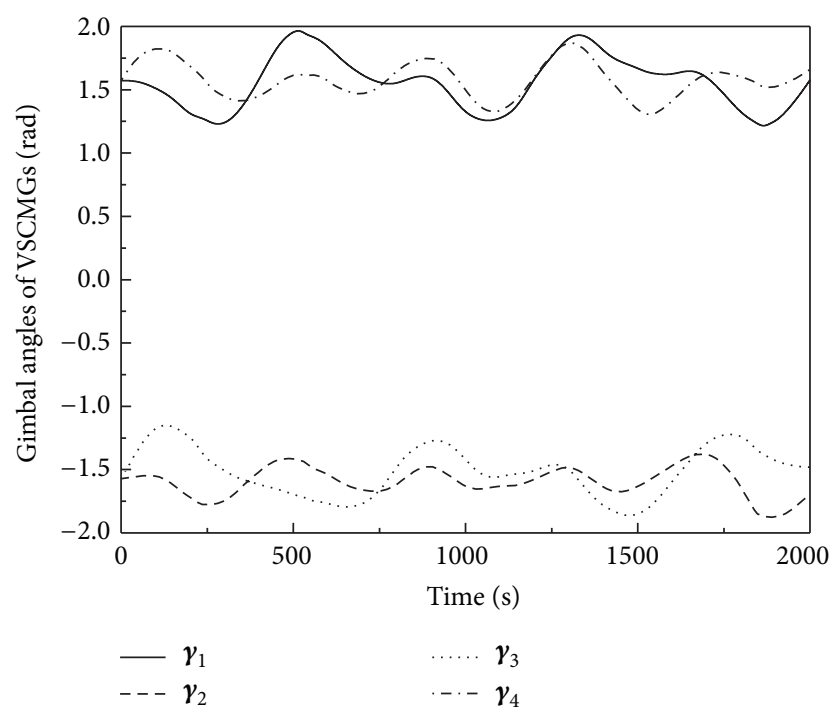

(a) With FADO

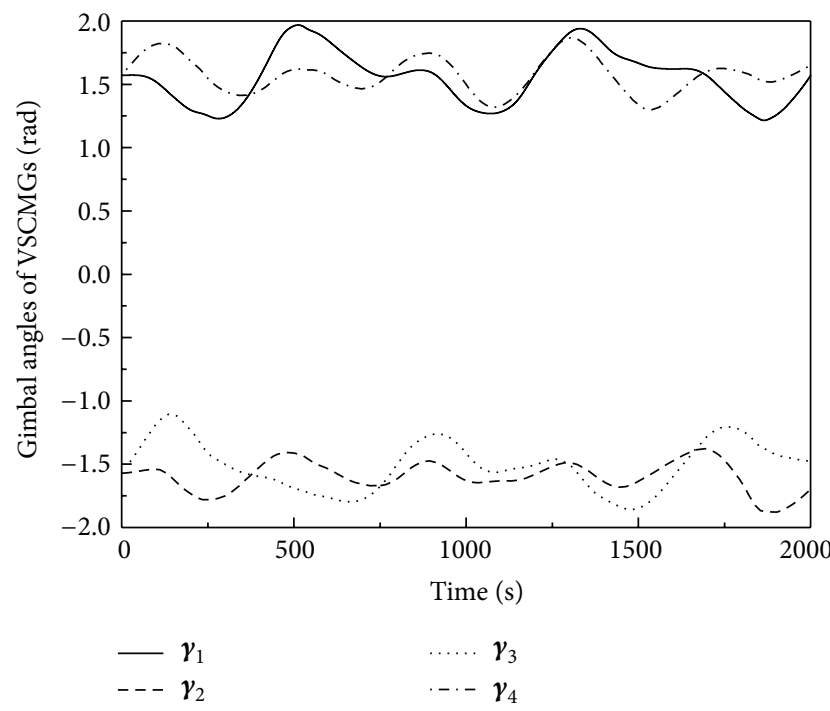

(b) Without a FADO

Figure 7: Comparison of the gimbal angles.

is capable of estimating the unknown nonlinearity of the spacecraft dynamics effectively, including the unknown mass moment in the inertia matrix, external disturbances, actuator friction, and an initial direction setting error, so the proposed controller can get accurately track attitude and power usage simultaneously.

\section{Conclusions}

This paper has proposed a novel algorithm for controlling the attitude tracking of spacecraft in the presence of an uncertain mass moment in the inertia matrix and external disturbances while simultaneously tracking a desired power profile using a cluster of VSCMGs. In the proposed control scheme, a FADO is used to estimate the unknown nonlinearity in the spacecraft dynamics online. In addition, the stability of the overall closed-loop system is guaranteed by the Lyapunov theory. The simulations demonstrate that the proposed controller can achieve the desired attitude tracking performance, even in the presence of an unknown mass moment in the inertia matrix, external disturbances, actuator friction, and an initial direction setting error. This paper suggests that the proposed controller can be developed for use in satellites to accurately track attitude and power usage by considering various factors that have been disregarded in previous studies. This paper details the process for developing 


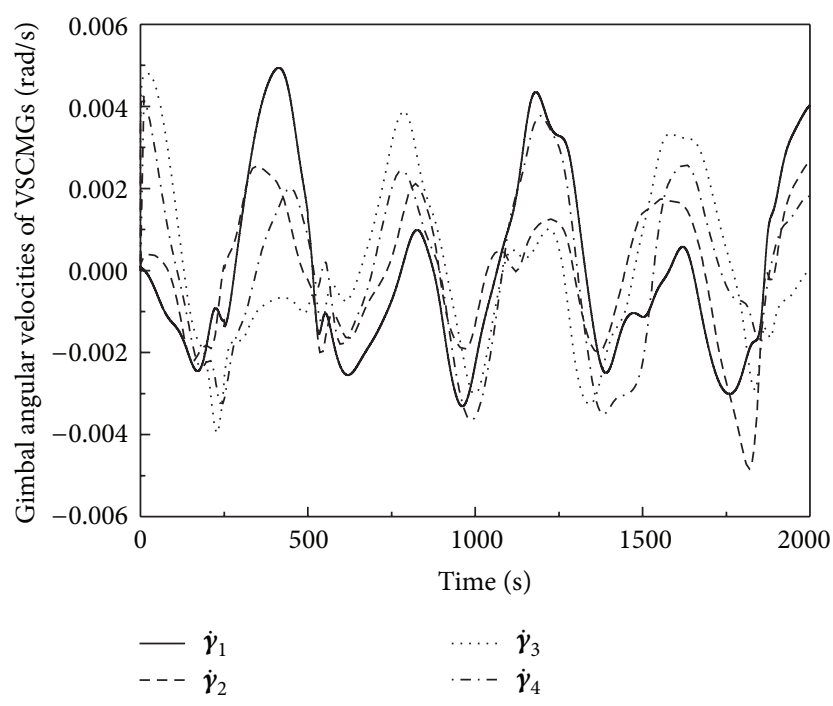

(a) With FADO

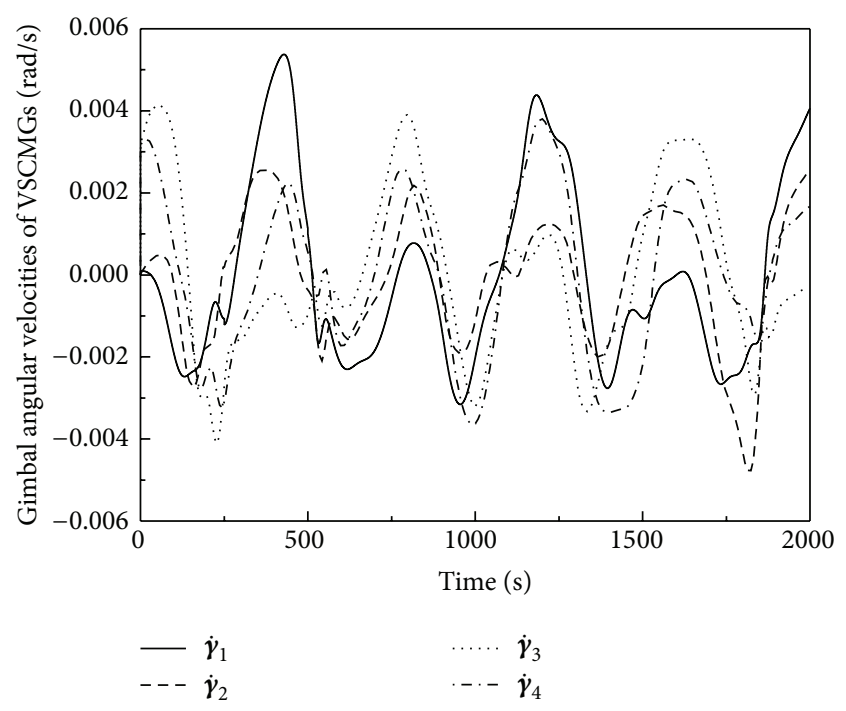

(b) Without a FADO

FIGURE 8: Comparison of the gimbal angular velocities.

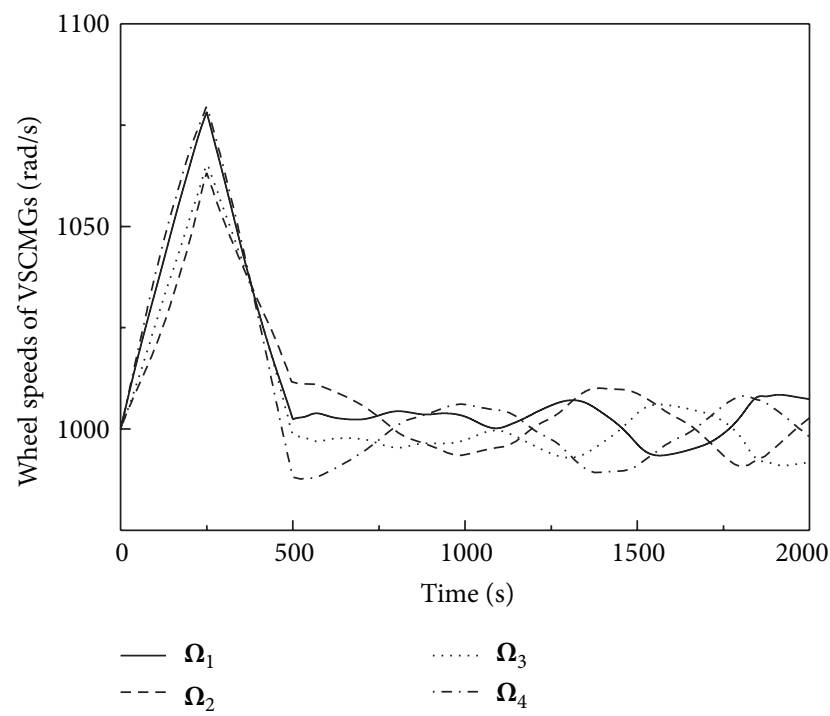

(a) With FADO

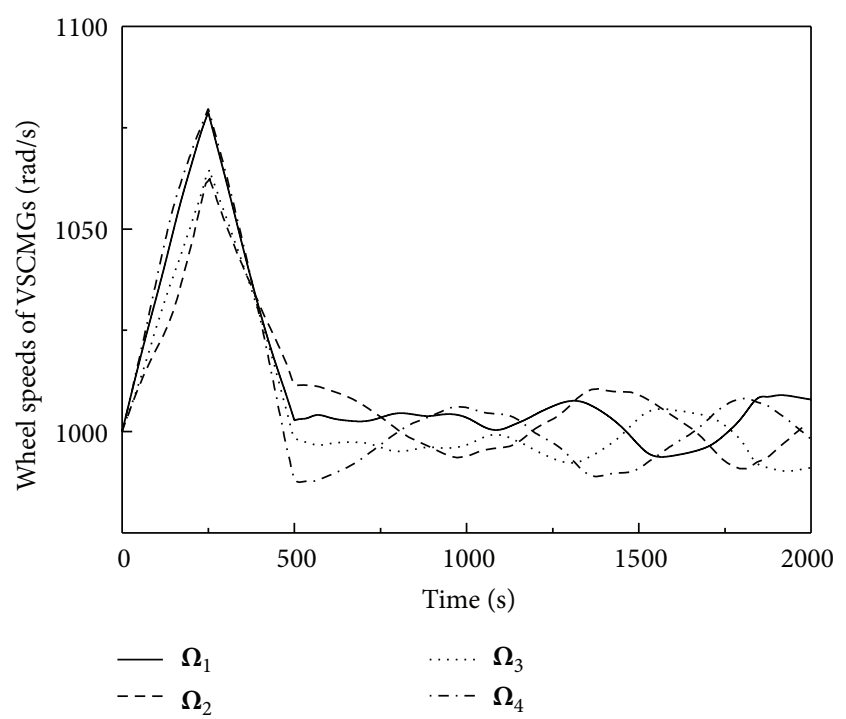

(b) Without a FADO

FIGURE 9: Comparison of the wheel speeds.

the controller by considering various environments in which the controller may be implemented. Future studies will focus on the simulation of an IPAC system installed on a satellite with variable configured deployable booms. We also intend to conduct a hardware-based demonstration of the IPAC approach with VSCMGs.

\section{Nomenclature}

J: Inertia matrix of the total spacecraft $\left(\mathrm{kg} \cdot \mathrm{m}^{2}\right)$
$\mathrm{J}_{0}$ : The combined matrix of the spacecraft and the point masses of the VSCMGs $\left(\mathrm{kg} \cdot \mathrm{m}^{2}\right)$

$\mathbf{I}_{g g}, \mathbf{I}_{g s}$, and $\mathbf{I}_{g t}$ : Inertia matrix of the gimbals of the VSCMGs, which correspond to the gimbal, spin, and transpose axis $\left(\mathrm{kg} \cdot \mathrm{m}^{2}\right)$

$\mathbf{I}_{w g}, \mathbf{I}_{w s}$, and $\mathbf{I}_{w t}$ : Inertia matrix of the wheels of the VSCMGs, which correspond to the gimbal, spin, and transpose axis $\left(\mathrm{kg} \cdot \mathrm{m}^{2}\right)$ 


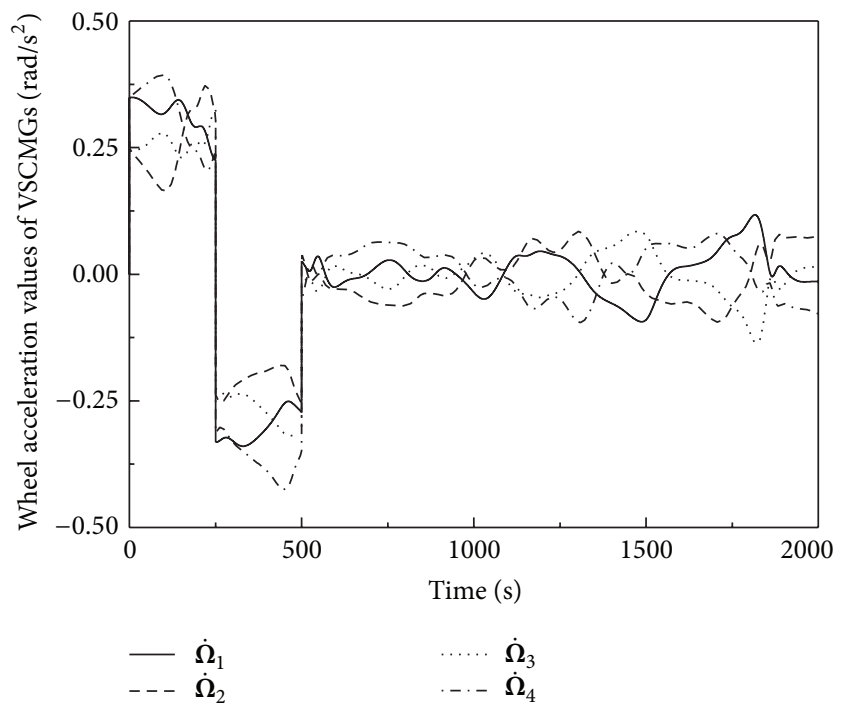

(a) With FADO

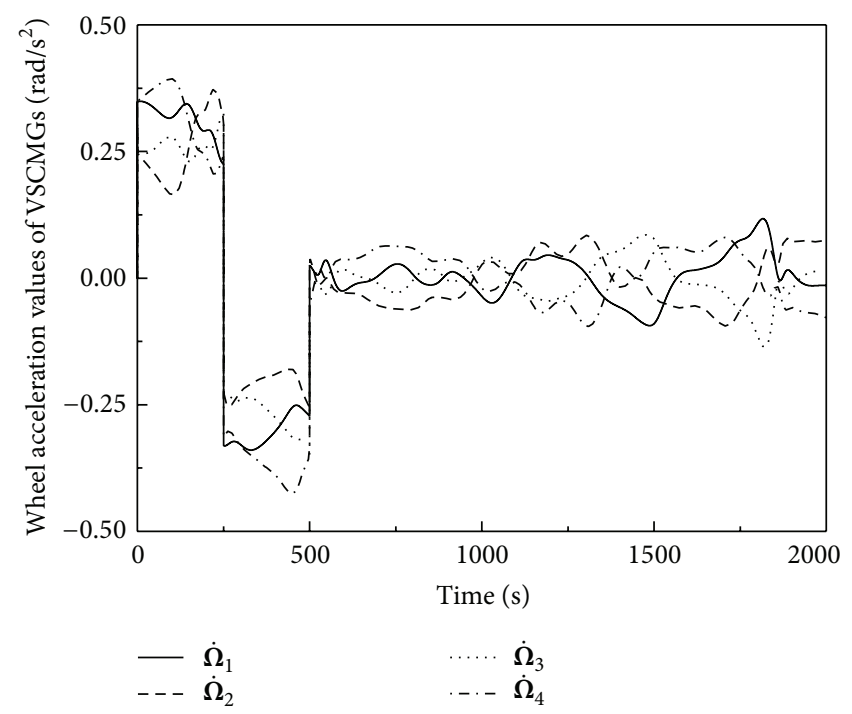

(b) Without a FADO

FIGURE 10: Comparison of the wheel acceleration values.

\footnotetext{
$\sigma: \quad$ Modified Rodrigues parameters

$\omega$ : Angular velocity of the spacecraft with respect to the inertia frame $(\mathrm{rad} / \mathrm{s})$

$\boldsymbol{\gamma}, \dot{\gamma}$ : Gimbal angle and gimbal angular velocity vector of the VSCMG ( $\mathrm{rad}, \mathrm{rad} / \mathrm{s})$

$\boldsymbol{\Omega}, \dot{\Omega}$ : Wheel speed and wheel acceleration vector of the VSCMG $\left(\mathrm{rad} / \mathrm{s}, \mathrm{rad} / \mathrm{s}^{2}\right)$

$\boldsymbol{\Omega}_{0}$ : Initial wheel speed vector of the VSCMG ( $\mathrm{rad} / \mathrm{s})$.
}

\section{Competing Interests}

The authors declare that there are no competing interests regarding the publication of this paper.

\section{Acknowledgments}

This work was jointly supported by the Natural Science Foundation of China Grants nos. 51375034 and 61327809.

\section{References}

[1] V. Lappas, D. Richie, C. Hall, J. Fausz, and B. Wilson, "Survey of technology developments in flywheel attitude control and energy storage systems," Journal of Guidance, Control, and Dynamics, vol. 32, no. 2, pp. 354-365, 2009.

[2] J. Fausz and B. DeKock, "Minimum resolvable torque mitigation using variable speed control moment gyros," in Proceedings of the AIAA Guidance, Navigation, and Control Conference, 20108070, Toronto, Canada, August 2010.

[3] D. J. Richie, V. J. Lappas, and B. Wie, "Practical steering law for small satellite energy storage and attitude control," Journal of
Guidance, Control, and Dynamics, vol. 32, no. 6, pp. 1898-1911, 2009.

[4] H. Yoon and P. Tsiotras, "Spacecraft adaptive attitude and power tracking with variable speed control moment gyroscopes," Journal of Guidance, Control, and Dynamics, vol. 25, no. 6, pp. 1081-1090, 2002.

[5] H. Yoon and P. Tsiotras, "Spacecraft line-of-sight control using a single variable-speed control moment gyro," Journal of Guidance, Control, and Dynamics, vol. 29, no. 6, pp. 1295-1308, 2006.

[6] J. Fan and D. Zhou, "Nonlinear attitude control of flexible spacecraft with scissored pairs of control moment gyros," Journal of Dynamic Systems, Measurement and Control, vol. 134, no. 5, Article ID 54502, 2012.

[7] B. T. Costic, D. M. Dawson, M. S. De Queiroz, and V. Kapila, "Quaternion-based adaptive attitude tracking controller without velocity measurements," Journal of Guidance, Control, and Dynamics, vol. 24, no. 6, pp. 1214-1222, 2001.

[8] Y. Xia, Z. Zhu, M. Fu, and S. Wang, "Attitude tracking of rigid spacecraft with bounded disturbances," IEEE Transactions on Industrial Electronics, vol. 58, no. 2, pp. 647-659, 2011.

[9] D. DeVon and R. Fuentes, "Adaptive attitude control and closedloop power tracking for an integrated power and attitude control system using variable speed control moment gyroscopes," in Proceedings of the AIAA Guidance, Navigation, and Control Conference and Exhibit, 2005-7080, San Francisco, Calif, USA, August 2005.

[10] M. Romano and B. N. Agrawal, "Attitude dynamics/control of dual-body spacecraft with variable speed control moment gyros," Journal of Guidance, Control, and Dynamics, vol. 27, no. 4, pp. 513-525, 2004.

[11] J. Park and A. Palazzolo, "Magnetically suspended VSCMGs for simultaneous attitude control and power transfer IPAC Service," Journal of Dynamic Systems, Measurement and Control, vol. 132, no. 5, Article ID 051001, 15 pages, 2010.

[12] D. Kim, W. MacKunis, N. Fitz-Coy, and W. E. Dixon, "Precision IPACS in the presence of dynamic uncertainty," in Proceedings of the IEEE Conference on Decision and Control, pp. 5959-5964, Shanghai, China, December 2009. 
[13] A.-M. Zou, K. D. Kumar, and Z.-G. Hou, "Quaternion-based adaptive output feedback attitude control of spacecraft using chebyshev neural networks," IEEE Transactions on Neural Networks, vol. 21, no. 9, pp. 1459-1471, 2010.

[14] V. S. Deshpande and S. B. Phadke, "Control of uncertain nonlinear systems using an uncertainty and disturbance estimator," Journal of Dynamic Systems, Measurement and Control, vol. 134, no. 2, Article ID 024501, 2012.

[15] W. MacKunis, K. Dupree, S. Bhasin, and W. E. Dixon, "Adaptive neural network satellite attitude control in the presence of inertia and CMG actuator uncertainties," in Proceedings of the American Control Conference, pp. 2975-2980, IEEE, Seattle, Wash, USA, June 2008.

[16] E. Sariyildiz and K. Ohnishi, "A guide to design disturbance observer," Journal of Dynamic Systems, Measurement, and Control, vol. 136, no. 2, Article ID 021011, 2014.

[17] A. H. El-Shaer, M. A. Janaideh, P. Krejci, and M. Tomizuka, "Robust performance enhancement using disturbance observers for hysteresis compensation based on generalized prandtl-ishlinskii model," Journal of Dynamic Systems, Measurement and Control, vol. 135, no. 5, Article ID 051008, 2013.

[18] Z. Chu, J. Cui, and F. Sun, "Fuzzy adaptive disturbanceobserver-based robust tracking control of electrically driven free-floating space manipulator," IEEE Systems Journal, vol. 8, no. 2, pp. 343-352, 2014.

[19] E. Kim and C. Park, "Fuzzy disturbance observer approach to robust tracking control of nonlinear sampled systems with the guaranteed suboptimal $H_{\infty}$ performance," IEEE Transactions on Systems, Man, and Cybernetics, Part B: Cybernetics, vol. 34, no. 3, pp. 1574-1581, 2004.

[20] M. B. Nazir and S. Wang, "Fine tuning of fuzzy rule-base system and rule set reduction using statistical analysis," Journal of Dynamic Systems, Measurement and Control, vol. 133, no. 4, Article ID 041003, 2011. 


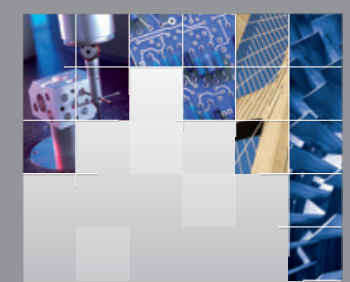

\section{Enfincering}
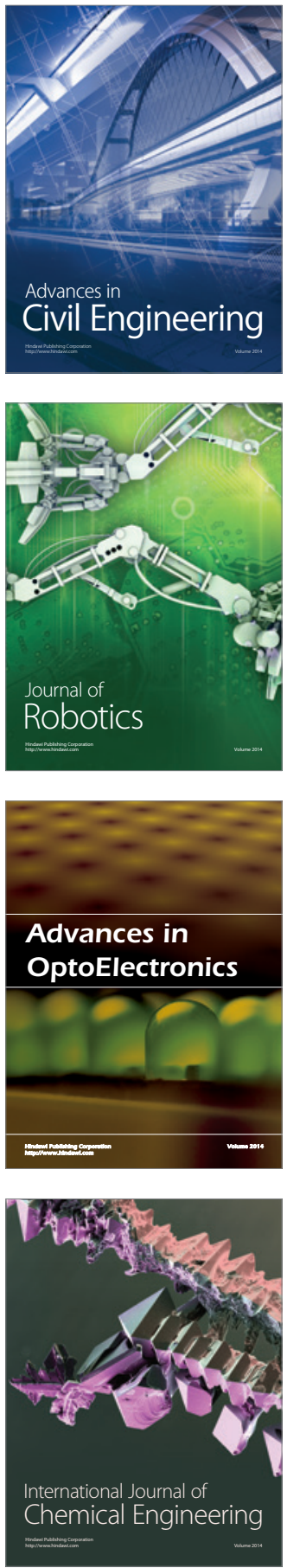

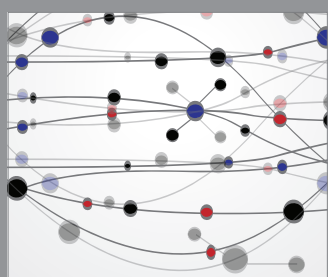

The Scientific World Journal

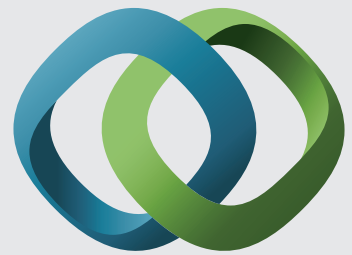

\section{Hindawi}

Submit your manuscripts at

http://www.hindawi.com
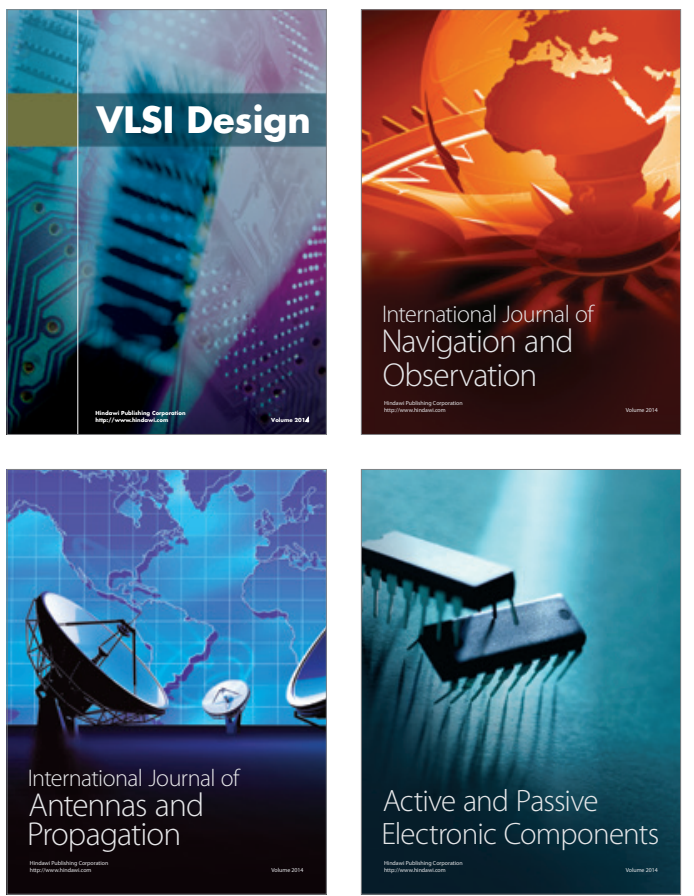
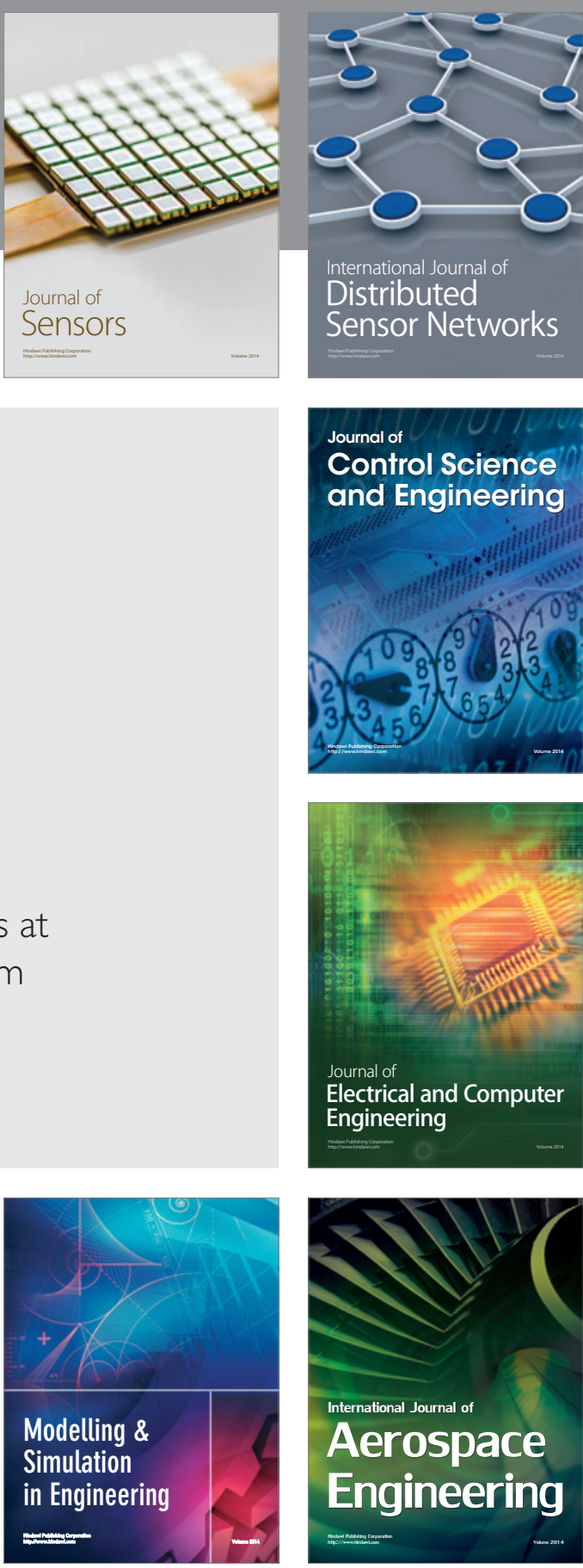

International Journal of

Distributed

Sensor Networks

Journal of

Control Science

and Engineering
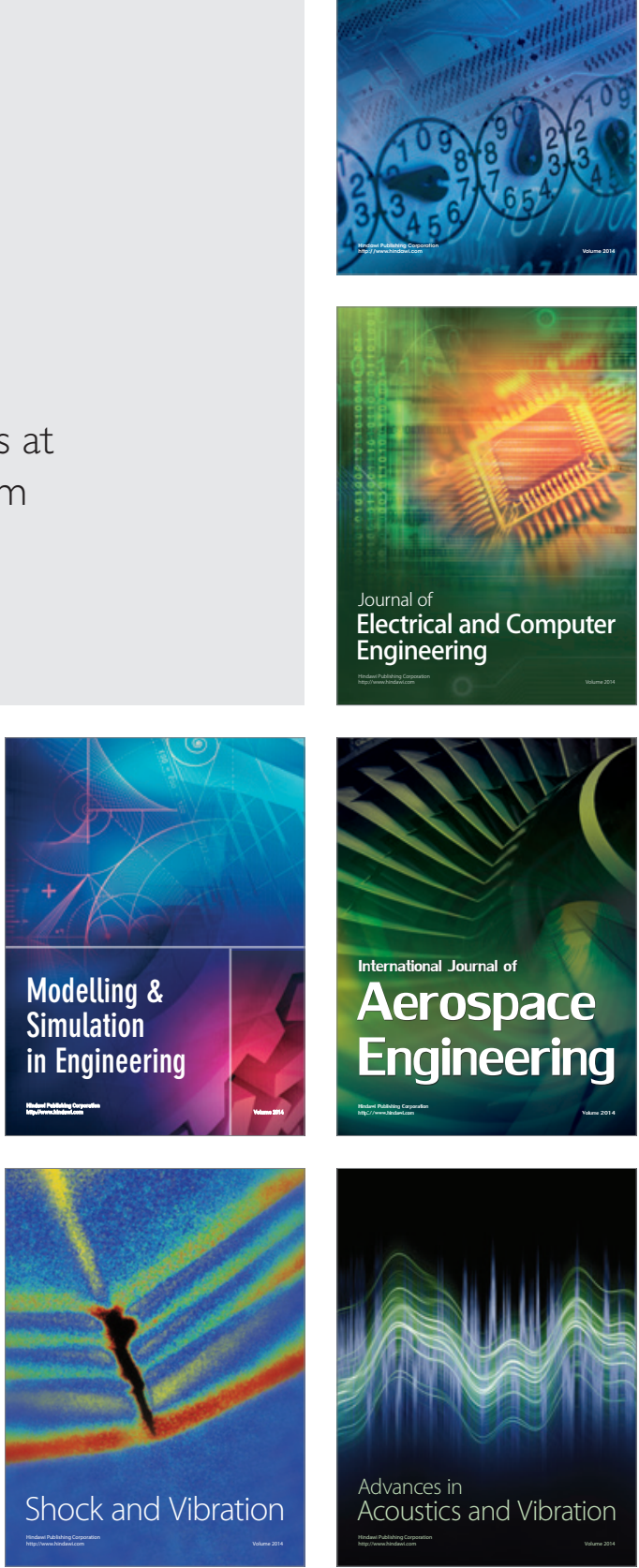